\title{
A survey of recent results on congruence lattices of lattices
}

\author{
JiŘí TŮmA AND FRIEDRICH WEHRUNG \\ Dedicated to Ralph McKenzie on his 60-th birthday
}

\begin{abstract}
We review recent results on congruence lattices of (infinite) lattices. We discuss results obtained with box products, as well as categorical, ring-theoretical, and topological results.
\end{abstract}

\section{Contents}

1. Introduction 1

2. Uniform Refinement Properties 3

3. The $M_{3}\langle L\rangle$ construction, tensor product, and box product 10

4. The functor $\mathrm{Con}_{\mathrm{c}}$ on partial lattices 14

5. Lifting diagrams of semilattices by diagrams of partial lattices 15

6. Extensions of partial lattices to lattices 18

7. Connections to ring theory 21

8. Dual topological spaces 23

9. Open problems 24

$\begin{array}{ll}\text { Acknowledgments } & 27\end{array}$

\section{Introduction}

For a lattice $L$, the congruence lattice of $L$, denoted here by $\operatorname{Con} L$, is the lattice of all congruences of $L$ under inclusion. As the congruence lattice of any algebraic system, the lattice Con $L$ is algebraic. The compact elements of Con $L$ are the finitely generated congruences, that is, the congruences of the form

$$
\bigvee_{i<n} \Theta_{L}\left(a_{i}, b_{i}\right)
$$

Date: October 1, 2002.

2000 Mathematics Subject Classification: 06B10, 06E05.

Key words and phrases: Lattice, congruence, box product, partial lattice, amalgamation, regular ring, locally matricial ring, dual topological space.

The first author was partially supported by GA UK grant no. 162/1999 and by GA CR grant no. 201/99. The second author was partially supported by the Fund of Mobility of the Charles University (Prague), by FRVS grant no. 2125, by institutional grant CEZ:J13/98:113200007, and by the Barrande program. 
where $n<\omega, a_{i}, b_{i} \in L$, for all $i<n$, and $\Theta_{L}\left(a_{i}, b_{i}\right)$ (the principal congruence generated by the pair $\left.\left\langle a_{i}, b_{i}\right\rangle\right)$ denotes the least congruence of $L$ that identifies $a_{i}$ and $b_{i}$. We denote by $\operatorname{Con}_{\mathrm{c}} L$, the congruence semilattice of $L$, the $\langle\vee, 0\rangle$-semilattice of all compact congruences of $L$. A classical result by N. Funayama and T. Nakayama [6] states that the lattice $\operatorname{Con} L$ is distributive. Hence the $\langle\vee, 0\rangle$-semilattice $\operatorname{Con}_{\mathrm{c}} L$ is distributive, that is, for all $\boldsymbol{a}, \boldsymbol{b}, \boldsymbol{c} \in \operatorname{Con}_{\mathrm{c}} L$, if $\boldsymbol{c} \leq \boldsymbol{a} \vee \boldsymbol{b}$, then there are elements $\boldsymbol{a}^{\prime} \leq \boldsymbol{a}$ and $\boldsymbol{b}^{\prime} \leq \boldsymbol{b}$ such that $\boldsymbol{c}=\boldsymbol{a}^{\prime} \vee \boldsymbol{b}^{\prime}$. Most of the concepts we shall use in the present paper are more conveniently expressed with Con $_{\mathrm{c}}$ than with Con.

Since the congruence lattice of any algebra is an algebraic lattice, it follows that the congruence lattice of any lattice is an algebraic distributive lattice. The question whether the converse of this result holds, that is, whether any algebraic distributive lattice is isomorphic to Con $L$, for some lattice $L$, has been raised in the early forties by R. P. Dilworth, who solved the finite case. We refer to this problem as the Congruence Lattice Problem, CLP in short. The semilattice formulation of CLP asks whether every distributive $\langle\vee, 0\rangle$-semilattice is isomorphic to $\operatorname{Con}_{\mathrm{c}} L$, for some lattice $L$.

Since the problem was raised, much progress has been done; we refer the reader to G. Grätzer and E. T. Schmidt [15] for a pre-1998 survey. Furthermore, it turns out that the topic of congruence lattices of lattices can be divided into two parts: congruence lattices of finite lattices, and congruence lattices of infinite lattices. These topics are nearly disjoint (surprisingly?), although there are a few noteworthy interactions between the two of them. We refer the reader to G. Grätzer and E. T. Schmidt [24] for a survey of congruence lattices of finite lattices.

About the infinite case, the last few years have seen the emergence of many new techniques and results about CLP that the present paper intends to survey. The main ideas can be separated into different groups.

- Uniform refinement properties (Section 2). Most known partial negative solutions to CLP are obtained via certain infinitary sentences of the theory of semilattices that hold in all semilattices of the form $\operatorname{Con}_{\mathrm{C}} L$, for $L$ in large classes of lattices, such as the class of relatively complemented lattices. On the other hand, these formulas do not hold in all distributive $\langle\vee, 0\rangle$-semilattices. It also turns out that all the presently known representation theorems yield semilattices with the strongest known 'uniform refinement property', which we denote here by $\mathrm{URP}^{+}$(see Propositions 2.10 and 2.11).

- The $M_{3}\langle L\rangle$ construction, tensor product, and box product (Section 3). It was proposed as an open problem in G. Grätzer and E. T. Schmidt [12], whether every nontrivial lattice has a proper congruencepreserving extension. A positive solution to this problem is presented in G. Grätzer and F. Wehrung [25]. The construction used there, the Boolean triple construction, as well as its generalization called the box product construction, see G. Grätzer and F. Wehrung [29], turned out to be very 
useful. In Section 3, we discuss some of the results that can be obtained with these constructions.

- Extending partial lattices to lattices (Sections 4, 5, and 6). The original Grätzer-Schmidt solution to the characterization problem of algebraic lattices as congruence lattices of algebras, see G. Grätzer and E. T. Schmidt [11], starts with a partial algebra that is further extended to a total algebra. However, this method requires to add infinitely many operations, thus it is, a priori, not suited for dealing with a class of objects of a fixed type such as lattices. However, there are some special methods that significantly extend the known positive results to wider classes of distributive $\langle\vee, 0\rangle$-semilattices.

- Ring-theoretical methods (Section 7). A survey of the connections between ring-theoretical problems and results and congruence lattice representation problems is presented in K. R. Goodearl and F. Wehrung [8]. In Section 7, we present a very short overview of the subject, as well as a few recent results.

- Finitely generated varieties of lattices (Section 8). It is proved in M. Ploščica, J. Tůma, and F. Wehrung [38] that for any nondistributive variety $\mathbf{V}$ of lattices and any set $X$ with at least $\aleph_{2}$ elements, the congruence lattice of the free lattice $\mathrm{F}_{\mathbf{V}}(X)$ satisfies many negative properties with respect to CLP, see Theorem 2.6; in particular, its semilattice of compact elements is not representable via Schmidt's Lemma (see E. T. Schmidt [41], Proposition 2.5, and Theorem 6.6) and it is not isomorphic to $\mathrm{Con}_{\mathrm{c}} L$, for any sectionally complemented lattice $L$. A variety of lattices is nondistributive iff it contains as an element either the diamond $M_{3}$ or the pentagon $N_{5}$. As a surprising consequence, even the very "simple" finitely generated lattice varieties $\mathbf{M}_{3}$ and $\mathbf{N}_{5}$ have complicated congruence classes (see Definition 8.1), not completely understood yet. Nevertheless, M. Ploščica's work is an important step in this direction.

\section{Uniform Refinement Properties}

The key to all known negative congruence lattice representation results lies in considering certain infinitary axioms of the theory of join-semilattices (we do not need the zero in their formulation) that we call uniform refinement properties. Although there is no precise definition of what a 'uniform refinement property' should be in general, the few of them that we shall review in this section undoubtedly offer a very recognizable pattern.

The first idea of this pattern can be found in the case of a finite number of equations (in a given join-semilattice), of the form

$$
\Sigma: \boldsymbol{a}_{i} \vee \boldsymbol{b}_{i}=\text { constant } \quad(\text { for all } i \in I)
$$




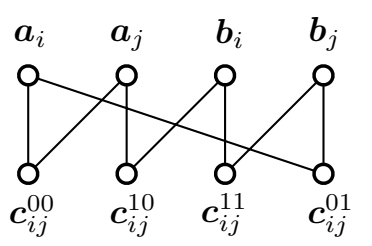

FIGURE 1. Refining $\boldsymbol{a}_{i} \vee \boldsymbol{b}_{i}=\boldsymbol{a}_{j} \vee \boldsymbol{b}_{j}$.

When $I=\{i, j\}$, a refinement of $\Sigma$ can be defined as a collection of four elements $c_{i j}^{u v}$ (for $u, v<2$ ) satisfying the equations

$$
\begin{aligned}
\boldsymbol{a}_{i}=\boldsymbol{c}_{i j}^{00} \vee \boldsymbol{c}_{i j}^{01} & \text { and } & \boldsymbol{b}_{i}=\boldsymbol{c}_{i j}^{10} \vee \boldsymbol{c}_{i j}^{11}, \\
\boldsymbol{a}_{j}=\boldsymbol{c}_{i j}^{00} \vee \boldsymbol{c}_{i j}^{10} & \text { and } & \boldsymbol{b}_{j}=\boldsymbol{c}_{i j}^{01} \vee \boldsymbol{c}_{i j}^{11},
\end{aligned}
$$

see Figure 1. Observe that (2.1) implies immediately the following:

$$
\boldsymbol{a}_{i} \leq \boldsymbol{a}_{j} \vee \boldsymbol{c}_{i j}^{01}
$$

When $I$ is an arbitrary finite set, with powerset $\mathcal{P}(I)$, one can extend this in a natural way and thus define a refinement of $\Sigma$ to be a $\mathcal{P}(I)$-indexed family of elements of $S$ satisfying suitable generalizations of (2.1). Nevertheless, this cannot be extended immediately to the infinite case, so that we shall focus instead on the consequence (2.2) of refinement, together with an additional "coherence condition" $\boldsymbol{c}_{i k}^{01} \leq \boldsymbol{c}_{i j}^{01} \vee \boldsymbol{c}_{j k}^{01}$. Thus we obtain the first uniform refinement property, see F. Wehrung [50]. This condition is a byproduct of a related infinitary axiom for dimension groups obtained in F. Wehrung [49]. Another (easily seen to be equivalent) form of this axiom, denoted by $\mathrm{URP}_{1}$ in J. Tůma and F. Wehrung [45], is the following, we shall denote it here by URP:

Definition 2.1. Let $S$ be a join-semilattice, let $\boldsymbol{e} \in S$. We say that $S$ satisfies URP at $\boldsymbol{e}$, if for all families $\left\langle\boldsymbol{a}_{i} \mid i \in I\right\rangle$ and $\left\langle\boldsymbol{b}_{i} \mid i \in I\right\rangle$ of elements of $S$ such that $\boldsymbol{a}_{i} \vee \boldsymbol{b}_{i}=\boldsymbol{e}$, for all $i \in I$, there are elements $\boldsymbol{a}_{i}^{*}, \boldsymbol{b}_{i}^{*}, \boldsymbol{c}_{i, j}$ (for $i, j \in I$ ) of $S$ such that the following statements hold:

(i) $\boldsymbol{a}_{i}^{*} \leq \boldsymbol{a}_{i}, \boldsymbol{b}_{i}^{*} \leq \boldsymbol{b}_{i}$, and $\boldsymbol{a}_{i}^{*} \vee \boldsymbol{b}_{i}^{*}=\boldsymbol{e}$, for all $i \in I$;

(ii) $\boldsymbol{c}_{i, j} \leq \boldsymbol{a}_{i}^{*}$ and $\boldsymbol{c}_{i, j} \leq \boldsymbol{b}_{j}^{*}$, for all $i, j \in I$;

(iii) $\boldsymbol{a}_{i}^{*} \leq \boldsymbol{a}_{j}^{*} \vee \boldsymbol{c}_{i, j}$ and $\boldsymbol{b}_{j}^{*} \leq \boldsymbol{b}_{i}^{*} \vee \boldsymbol{c}_{i, j}$, for all $i, j \in I$;

(iv) $\boldsymbol{c}_{i, k} \leq \boldsymbol{c}_{i, j} \vee \boldsymbol{c}_{j, k}$, for all $i, j, k \in I$.

We say that $S$ has URP, if it has URP at all its elements.

A slight weakening of URP is introduced in M. Ploščica, J. Tůma, and F. Wehrung [38]:

Definition 2.2. Let $S$ be a join-semilattice, let $e \in S$. We say that $S$ satisfies WURP at $\boldsymbol{e}$, if for all families $\left\langle\boldsymbol{a}_{i} \mid i \in I\right\rangle$ and $\left\langle\boldsymbol{b}_{i} \mid i \in I\right\rangle$ of elements of $S$ such that $\boldsymbol{a}_{i} \vee \boldsymbol{b}_{i}=\boldsymbol{e}$, for all $i \in I$, there are elements $\boldsymbol{c}_{i, j}$ (for $i, j \in I$ ) of $S$ such that the following statements hold:

(i) $\boldsymbol{c}_{i, j} \leq \boldsymbol{a}_{i}, \boldsymbol{b}_{j}$, for all $i, j \in I$; 
(ii) $\boldsymbol{a}_{j} \vee \boldsymbol{b}_{i} \vee \boldsymbol{c}_{i, j}=\boldsymbol{e}$, for all $i, j \in I$;

(iii) $\boldsymbol{c}_{i, k} \leq \boldsymbol{c}_{i, j} \vee \boldsymbol{c}_{j, k}$, for all $i, j, k \in I$.

We say that $S$ has WURP, if it has WURP at all its elements.

Definition 2.3. Let $S$ and $T$ be join-semilattices, let $\boldsymbol{e} \in S$. A join-homomorphism $\mu: S \rightarrow T$ is weakly distributive, if for all $\boldsymbol{a}, \boldsymbol{b} \in T$ such that $\mu(\boldsymbol{e})=\boldsymbol{a} \vee \boldsymbol{b}$, there are $\boldsymbol{a}^{\prime}, \boldsymbol{b}^{\prime} \in S$ such that $\mu\left(\boldsymbol{a}^{\prime}\right) \leq \boldsymbol{a}, \mu\left(\boldsymbol{b}^{\prime}\right) \leq \boldsymbol{b}$, and $\boldsymbol{a}^{\prime} \vee \boldsymbol{b}^{\prime}=\boldsymbol{e}$.

For further use (see Theorem 6.6), we record here the following definition:

Definition 2.4. Let $\boldsymbol{a}$ be a congruence of a join-semilattice $S$. We say that $\boldsymbol{a}$ is

- weakly distributive, if the canonical projection from $S$ onto $S / \boldsymbol{a}$ is weakly distributive;

- monomial, if every congruence class of $\boldsymbol{a}$ has a largest element;

- distributive, if $\boldsymbol{a}$ is a union of a family of weakly distributive and monomial congruences of $S$.

For join-semilattices $S$ and $T$, a surjective join-homomorphism $\mu: S \rightarrow T$ is distributive, if its kernel is a distributive congruence of $S$.

The following easy result records standard facts about uniform refinement properties and weakly distributive homomorphisms, see M. Ploščica, J. Tůma, and F. Wehrung [38] and F. Wehrung [50]:

Proposition 2.5. Let $S$ and $T$ be join-semilattices. Then the following statements hold:

(i) If $S$ is distributive, then the set of all elements of $S$ at which URP (resp., WURP) holds is closed under finite joins.

(ii) For any weakly distributive join-homomorphism $\mu: S \rightarrow T$ (see Definition 2.3) and any $\boldsymbol{e} \in S$, if $S$ has URP (resp., WURP) at $\boldsymbol{e}$, then $T$ has URP (resp., WURP) at $\mu(\boldsymbol{e})$.

(iii) URP implies WURP.

The following result, obtained in M. Ploščica, J. Tůma, and F. Wehrung [38] and in J. Tůma and F. Wehrung [45], explains how uniform refinement properties can be used to separate classes of semilattices:

\section{Theorem 2.6.}

(i) For every lattice $L$ with permutable congruences, the congruence semilattice $\mathrm{Con}_{\mathrm{c}} L$ satisfies URP.

(ii) Let $\mathbf{V}$ be a nondistributive variety of lattices, let $X$ be a set with at least $\aleph_{2}$ elements. Then $\operatorname{Con}_{\mathrm{c}} \mathrm{F}_{\mathbf{V}}(X)$ does not satisfy WURP.

Although Theorem 2.6 is a difficult result, its set-theoretical part, that explains what is so special about the cardinality $\aleph_{2}$, is a very simple statement of infinite combinatorics, see C. Kuratowski [34]:

The Kuratowski Free Set Theorem. Let $n$ be a positive integer, let $X$ be a set. Then $|X| \geq \aleph_{n}$ iff for every map $\Phi:[X]^{n} \rightarrow[X]^{<\omega}$, there exists $U \in[X]^{n+1}$ such that $u \notin \Phi(U \backslash\{u\})$, for any $u \in U$. 
In fact, only the case $n=2$ is used.

A more complicated weakening of URP is used in J. Ti̊ma and F. Wehrung [45]:

Definition 2.7. Let $S$ be a join-semilattice, let $\boldsymbol{e} \in S$. We say that $S$ satisfies $\mathrm{URP}^{-}$at $\boldsymbol{e}$, if for all families $\left\langle\boldsymbol{a}_{i} \mid i \in I\right\rangle$ and $\left\langle\boldsymbol{b}_{i} \mid i \in I\right\rangle$ of elements of $S$ such that $\boldsymbol{a}_{i} \vee \boldsymbol{b}_{i}=\boldsymbol{e}$, for all $i \in I$, there are a subset $X$ of $I$ and elements $\boldsymbol{a}_{i}^{*}, \boldsymbol{b}_{i}^{*}, \boldsymbol{c}_{i, j}$ (for $i$, $j \in I$ ) of $S$ such that the following statements hold:

(i) $\boldsymbol{a}_{i}^{*} \leq \boldsymbol{a}_{i}, \boldsymbol{b}_{i}^{*} \leq \boldsymbol{b}_{i}$, and $\boldsymbol{a}_{i}^{*} \vee \boldsymbol{b}_{i}^{*}=\boldsymbol{e}$, for all $i \in I$;

(ii) $\boldsymbol{c}_{i, j} \leq \boldsymbol{a}_{i}^{*}$ and $\boldsymbol{c}_{i, j} \leq \boldsymbol{b}_{j}^{*}$, for all $i, j \in I$;

(iii) $\boldsymbol{a}_{i}^{*} \leq \boldsymbol{a}_{j}^{*} \vee \boldsymbol{c}_{i, j}$ and $\boldsymbol{b}_{j}^{*} \leq \boldsymbol{b}_{i}^{*} \vee \boldsymbol{c}_{i, j}$, for all $i, j \in I$;

(iv) $\boldsymbol{c}_{i, k} \leq \boldsymbol{c}_{i, j} \vee \boldsymbol{c}_{j, k}$, for all $i, j, k \in I$ such that the following two conditions hold:

$$
\begin{aligned}
& i, k \in X \text { implies that } j \in X, \\
& i, k \notin X \text { implies that } j \notin X .
\end{aligned}
$$

We say that $S$ has $\mathrm{URP}^{-}$, if it has $\mathrm{URP}^{-}$at all its elements.

The following difficult result, see J. Ti̊ma and F. Wehrung [45], extends Theorem 2.6:

\section{Theorem 2.8.}

(i) For every lattice $L$ with almost permutable congruences, the congruence semilattice $\mathrm{Con}_{\mathrm{c}} L$ satisfies $\mathrm{URP}^{-}$at every principal congruence of $L$.

(ii) Let $\mathbf{V}$ be a nondistributive variety of lattices, let $X$ be a set with at least $\aleph_{2}$ elements. Then the congruence semilattice of the $\mathbf{V}$-free bounded lattice $\mathrm{FB}_{\mathbf{V}}(X)$ over $X$ does not satisfy $\mathrm{URP}^{-}$at the largest congruence of $\mathrm{FB}_{\mathbf{V}}(X)$.

We end this section by presenting the following strengthening of URP:

Definition 2.9. Let $S$ be a join-semilattice, let $\boldsymbol{e} \in S$. We say that $S$ satisfies $\mathrm{URP}^{+}$at $\boldsymbol{e}$, if for all families $\left\langle\boldsymbol{a}_{i} \mid i \in I\right\rangle$ and $\left\langle\boldsymbol{b}_{i} \mid i \in I\right\rangle$ of elements of $S$ such that $\boldsymbol{a}_{i} \vee \boldsymbol{b}_{i}=\boldsymbol{e}$, for all $i \in I$, there are elements $\boldsymbol{c}_{i, j}$ (for $i, j \in I$ ) of $S$ such that the following statements hold:

(i) $\boldsymbol{c}_{i, j} \leq \boldsymbol{a}_{i}$ and $\boldsymbol{c}_{i, j} \leq \boldsymbol{b}_{j}$, for all $i, j \in I$;

(ii) $\boldsymbol{a}_{i} \leq \boldsymbol{a}_{j} \vee \boldsymbol{c}_{i, j}$ and $\boldsymbol{b}_{j} \leq \boldsymbol{b}_{i} \vee \boldsymbol{c}_{i, j}$, for all $i, j \in I$;

(iii) $\boldsymbol{c}_{i, k} \leq \boldsymbol{c}_{i, j} \vee \boldsymbol{c}_{j, k}$, for all $i, j, k \in I$.

We say that $S$ has $\mathrm{URP}^{+}$, if it has $\mathrm{URP}^{+}$at all its elements.

In view of Corollary 6.7 , it follows that all known recent representation theorems yield semilattices with $\mathrm{URP}^{+}$:

Proposition 2.10. The congruence semilattice $\mathrm{Con}_{\mathrm{c}} L$ satisfies $\mathrm{URP}^{+}$, for any relatively complemented lattice $L$.

Proof. Let $\boldsymbol{e} \in \operatorname{Con}_{\mathrm{C}} L$, let $\left\langle\boldsymbol{a}_{i} \mid i \in I\right\rangle$ and $\left\langle\boldsymbol{b}_{i} \mid i \in I\right\rangle$ be families of elements of $\mathrm{Con}_{\mathrm{C}} L$ such that $\boldsymbol{a}_{i} \vee \boldsymbol{b}_{i}=\boldsymbol{e}$, for all $i \in I$, we shall find elements $\boldsymbol{c}_{i, j}$ (for all $i, j \in I$ ) of $\mathrm{Con}_{\mathrm{c}} L$ that satisfy the required inequalities. Since $L$ is relatively 
complemented, every compact congruence of $L$ is principal, thus there are elements $u \leq v$ in $L$ such that $\boldsymbol{e}=\Theta(u, v)$.

Furthermore, it follows from Proposition 3.2 in F. Wehrung [50] that $L$ is "congruence splitting", thus, for all $i \in I$, since $\boldsymbol{a}_{i} \vee \boldsymbol{b}_{i}=\boldsymbol{e}$, there are $x_{i}, y_{i} \in[u, v]$ such that $x_{i} \vee y_{i}=v, \Theta\left(u, x_{i}\right) \subseteq \boldsymbol{a}_{i}$, and $\Theta\left(u, y_{i}\right) \subseteq \boldsymbol{b}_{i}$.

Since $\boldsymbol{a}_{i}$ is a compact congruence of $L$, it is principal, thus we can write $\boldsymbol{a}_{i}=$ $\Theta\left(u_{i}, v_{i}\right)$, for some $u_{i} \leq v_{i}$ in $L$, so $\Theta\left(u_{i}, v_{i}\right) \subseteq \Theta(u, v)$. Thus there exists a subdivision of the interval $\left[u_{i}, v_{i}\right]$ whose subintervals are all weakly project into $[u, v]$ (see Theorem III.1.2 in G. Grätzer [9]). Hence, since $L$ is relatively complemented, any of these intervals is projective to a subinterval of $[u, v]$ (see Exercise III.1.3 in G. Grätzer [9]), thus (again since $L$ is relatively complemented) to an interval of the form $[u, w]$, where $u \leq w \leq v$. Denoting by $s_{i}$ the join of all the $w$-s thus obtained; we get that $u \leq s_{i} \leq v$, while $\boldsymbol{a}_{i}=\Theta\left(u, s_{i}\right)$. Similarly, we can get $t_{i} \in[u, v]$ such that $\boldsymbol{b}_{i}=\Theta\left(u, t_{i}\right)$. Define $a_{i}=x_{i} \vee s_{i}$ and $b_{i}=y_{i} \vee t_{i}$. The relevant properties of $a_{i}$ and $b_{i}$ are the following:

$$
a_{i}, b_{i} \in[u, v] ; \quad a_{i} \vee b_{i}=v ; \quad \boldsymbol{a}_{i}=\Theta\left(u, a_{i}\right) ; \quad \boldsymbol{b}_{i}=\Theta\left(u, b_{i}\right) .
$$

We define compact congruences of $L$ by

$$
\boldsymbol{c}_{i, j}^{\prime}=\Theta^{+}\left(a_{i}, a_{j}\right) \quad \text { and } \quad \boldsymbol{c}_{i, j}^{\prime \prime}=\Theta^{+}\left(b_{j}, b_{i}\right), \quad \text { for all } i, j \in I,
$$

where we define $\Theta^{+}(x, y)=\Theta(x \wedge y, x)$, see Section 4. For all $i, j, k \in I$, it is not hard to verify the following inequalities:

$$
\begin{array}{lll}
\boldsymbol{c}_{i, j}^{\prime} \subseteq \boldsymbol{a}_{i}, \boldsymbol{b}_{j} ; & \boldsymbol{a}_{i} \subseteq \boldsymbol{a}_{j} \vee \boldsymbol{c}_{i, j}^{\prime} ; & \boldsymbol{c}_{i, k}^{\prime} \subseteq \boldsymbol{c}_{i, j}^{\prime} \vee \boldsymbol{c}_{j, k}^{\prime} ; \\
\boldsymbol{c}_{i, j}^{\prime \prime} \subseteq \boldsymbol{a}_{i}, \boldsymbol{b}_{j} ; & \boldsymbol{b}_{j} \subseteq \boldsymbol{b}_{i} \vee \boldsymbol{c}_{i, j}^{\prime \prime} ; & \boldsymbol{c}_{i, k}^{\prime \prime} \subseteq \boldsymbol{c}_{i, j}^{\prime \prime} \vee \boldsymbol{c}_{j, k}^{\prime \prime} .
\end{array}
$$

For example, for any congruence $\boldsymbol{e}$ of $L$, if $\boldsymbol{b}_{j} \subseteq \boldsymbol{e}$, that is, $b_{j} \equiv_{\boldsymbol{e}} u$, then, by the first two equations of $(2.3), a_{j} \equiv_{\boldsymbol{e}} v$, whence $a_{i} \leq_{\boldsymbol{e}} a_{j}$, that is, $\boldsymbol{c}_{i, j}^{\prime} \subseteq \boldsymbol{b}_{j}$. Therefore, by putting $\boldsymbol{c}_{i, j}=\boldsymbol{c}_{i, j}^{\prime} \vee \boldsymbol{c}_{i, j}^{\prime \prime}$, for all $i, j \in I$, we obtain the following inequalities

$$
\boldsymbol{c}_{i, j} \subseteq \boldsymbol{a}_{i}, \boldsymbol{b}_{j} ; \quad \boldsymbol{a}_{i} \subseteq \boldsymbol{a}_{j} \vee \boldsymbol{c}_{i, j} ; \quad \boldsymbol{b}_{j} \subseteq \boldsymbol{b}_{i} \vee \boldsymbol{c}_{i, j} ; \quad \boldsymbol{c}_{i, k} \subseteq \boldsymbol{c}_{i, j} \vee \boldsymbol{c}_{j, k},
$$

which concludes the proof.

We also refer the reader to Problem 9.

We can also prove the following result (compare it with Theorem 6.5(i)):

Proposition 2.11. Any $\vee$-direct limit over a totally ordered set of distributive lattices satisfies $\mathrm{URP}^{+}$.

Proof. Let $S$ be a $\vee$-direct limit over a totally ordered set $\theta$ of distributive lattices, say, $S=\varliminf_{\beta} \varliminf_{\theta \in} S_{\alpha}$ of distributive lattices $S_{\alpha}$, with transition maps $f_{\alpha, \beta}: S_{\alpha} \rightarrow S_{\beta}$, for $\alpha \leq \beta$ in $\theta$ and limiting maps $f_{\alpha}: S_{\alpha} \rightarrow S$, for $\alpha \in \theta$ (so the $f_{\alpha, \beta^{-S}}$ and the $f_{\alpha}$-s are join-homomorphisms). We prove that $S$ satisfies $\mathrm{URP}^{+}$. By extracting from $\theta$ a cofinal well-ordered chain, we may assume, without loss of generality, that $\theta$ is an ordinal. Let $\boldsymbol{e} \in S$, let $\left\langle\boldsymbol{a}_{i} \mid i \in I\right\rangle$ and $\left\langle\boldsymbol{b}_{i} \mid i \in I\right\rangle$ be families of elements of $S$ such that $\boldsymbol{a}_{i} \vee \boldsymbol{b}_{i}=\boldsymbol{e}$, for all $i \in I$; we shall find elements $\boldsymbol{c}_{i, j}$ (for $i, j \in I$ ) of $S$ that satisfy the required inequalities. 
Without loss of generality, $\boldsymbol{e}$ belongs to the range of $f_{0}$, so $\boldsymbol{e}=f_{0}\left(\boldsymbol{e}^{0}\right)$, for some $\boldsymbol{e}^{0} \in S_{0}$. Put $\boldsymbol{e}^{\alpha}=f_{0, \alpha}\left(\boldsymbol{e}^{0}\right)$, for all $\alpha<\theta$; observe that $f_{\alpha}\left(\boldsymbol{e}^{\alpha}\right)=\boldsymbol{e}$.

For all $i \in I$, there are $\alpha<\theta$ and $\boldsymbol{u}, \boldsymbol{v} \in S_{\alpha}$ such that $\boldsymbol{a}_{i}=f_{\alpha}(\boldsymbol{u}), \boldsymbol{b}_{i}=f_{\alpha}(\boldsymbol{v})$, and $\boldsymbol{u} \vee \boldsymbol{v}=\boldsymbol{e}^{\alpha}$. Denote by $\mu(i)$ the least such $\alpha$, and let $\left\langle\boldsymbol{a}_{i}^{\mu(i)}, \boldsymbol{b}_{i}^{\mu(i)}\right\rangle$ be a corresponding choice for $\langle\boldsymbol{u}, \boldsymbol{v}\rangle$. Put $\boldsymbol{a}_{i}^{\alpha}=f_{\mu(i), \alpha}\left(\boldsymbol{a}_{i}^{\mu(i)}\right)$ and $\boldsymbol{b}_{i}^{\alpha}=f_{\mu(i), \alpha}\left(\boldsymbol{b}_{i}^{\mu(i)}\right)$, for all $\alpha<\theta$ with $\alpha \geq \mu(i)$. Further, define $\nu(i, j)=\max \{\mu(i), \mu(j)\}$, for all $i, j \in I$.

We denote by $\wedge_{\alpha}$ the meet operation in $S_{\alpha}$, for all $\alpha<\theta$, and for all $i, j \in I$, we define

$$
\boldsymbol{c}_{i, j}=f_{\nu(i, j)}\left(\boldsymbol{a}_{i}^{\nu(i, j)} \wedge_{\nu(i, j)} \boldsymbol{b}_{j}^{\nu(i, j)}\right) .
$$

To conclude the proof, if suffices to establish that the elements $\boldsymbol{c}_{i, j}$ thus defined satisfy the required inequalities.

Let $i, j, k \in I$, set $\alpha=\nu(i, j), \beta=\nu(j, k)$, and $\gamma=\nu(i, k)$. We observe that the following inequality holds:

$$
\gamma \leq \max \{\alpha, \beta\}
$$

We first observe that $\boldsymbol{c}_{i, j} \leq f_{\alpha}\left(\boldsymbol{a}_{i}^{\alpha}\right)=\boldsymbol{a}_{i}$, and, similarly, $\boldsymbol{c}_{i, j} \leq \boldsymbol{b}_{j}$.

We further compute:

$$
\begin{aligned}
\boldsymbol{a}_{i}^{\alpha} & =\boldsymbol{a}_{i}^{\alpha} \wedge_{\alpha} \boldsymbol{e}^{\alpha} \\
& =\boldsymbol{a}_{i}^{\alpha} \wedge_{\alpha}\left(\boldsymbol{a}_{j}^{\alpha} \vee \boldsymbol{b}_{j}^{\alpha}\right) \\
& \left.=\left(\boldsymbol{a}_{i}^{\alpha} \wedge_{\alpha} \boldsymbol{a}_{j}^{\alpha}\right) \vee\left(\boldsymbol{a}_{i}^{\alpha} \wedge_{\alpha} \boldsymbol{b}_{j}^{\alpha}\right) \quad \text { (by the distributivity of } S_{\alpha}\right) \\
& \leq \boldsymbol{a}_{j}^{\alpha} \vee\left(\boldsymbol{a}_{i}^{\alpha} \wedge_{\alpha} \boldsymbol{b}_{j}^{\alpha}\right),
\end{aligned}
$$

whence, by applying $f_{\alpha}$, we obtain that $\boldsymbol{a}_{i} \leq \boldsymbol{a}_{j} \vee \boldsymbol{c}_{i, j}$. The proof of $\boldsymbol{b}_{j} \leq \boldsymbol{b}_{i} \vee \boldsymbol{c}_{i, j}$ is similar.

Finally, we verify the inequality

$$
\boldsymbol{c}_{i, k} \leq \boldsymbol{c}_{i, j} \vee \boldsymbol{c}_{j, k}
$$

We separate cases.

Case 1. $\alpha \leq \beta$. It follows from (2.4) that $\gamma \leq \beta$ as well. We establish further inequalities. We begin with the following:

$$
f_{\gamma, \beta}\left(\boldsymbol{a}_{i}^{\gamma} \wedge_{\gamma} \boldsymbol{b}_{k}^{\gamma}\right) \leq f_{\alpha, \beta}\left(\boldsymbol{a}_{i}^{\alpha} \wedge_{\alpha} \boldsymbol{b}_{j}^{\alpha}\right) \vee \boldsymbol{a}_{j}^{\beta}
$$

Indeed, $f_{\gamma, \beta}\left(\boldsymbol{a}_{i}^{\gamma} \wedge_{\gamma} \boldsymbol{b}_{k}^{\gamma}\right) \leq f_{\gamma, \beta}\left(\boldsymbol{a}_{i}^{\gamma}\right)=\boldsymbol{a}_{i}^{\beta}=f_{\alpha, \beta}\left(\boldsymbol{a}_{i}^{\alpha}\right)$ and $\boldsymbol{a}_{j}^{\beta}=f_{\alpha, \beta}\left(\boldsymbol{a}_{j}^{\alpha}\right)$, thus, in order to prove (2.6), it suffices to verify that $\boldsymbol{a}_{i}^{\alpha} \leq\left(\boldsymbol{a}_{i}^{\alpha} \wedge_{\alpha} \boldsymbol{b}_{j}^{\alpha}\right) \vee \boldsymbol{a}_{j}^{\alpha}$, which holds by the distributivity of $S_{\alpha}$ since $\boldsymbol{a}_{j}^{\alpha} \vee \boldsymbol{b}_{j}^{\alpha}=\boldsymbol{e}^{\alpha}$.

Next, we prove the following inequality:

$$
f_{\gamma, \beta}\left(\boldsymbol{a}_{i}^{\gamma} \wedge_{\gamma} \boldsymbol{b}_{k}^{\gamma}\right) \leq f_{\alpha, \beta}\left(\boldsymbol{a}_{i}^{\alpha} \wedge_{\alpha} \boldsymbol{b}_{j}^{\alpha}\right) \vee \boldsymbol{b}_{k}^{\beta}
$$

Indeed, $f_{\gamma, \beta}\left(\boldsymbol{a}_{i}^{\gamma} \wedge_{\gamma} \boldsymbol{b}_{k}^{\gamma}\right) \leq f_{\gamma, \beta}\left(\boldsymbol{b}_{k}^{\gamma}\right)=\boldsymbol{b}_{k}^{\beta} \leq f_{\alpha, \beta}\left(\boldsymbol{a}_{i}^{\alpha} \wedge_{\alpha} \boldsymbol{b}_{j}^{\alpha}\right) \vee \boldsymbol{b}_{k}^{\beta}$. Therefore, by using the distributivity of $S_{\beta}$ and the inequalities (2.6) and (2.7), we obtain the following 
inequalities:

$$
\begin{aligned}
\boldsymbol{c}_{i, j} \vee \boldsymbol{c}_{j, k} & =f_{\alpha}\left(\boldsymbol{a}_{i}^{\alpha} \wedge_{\alpha} \boldsymbol{b}_{j}^{\alpha}\right) \vee f_{\beta}\left(\boldsymbol{a}_{j}^{\beta} \wedge_{\beta} \boldsymbol{b}_{k}^{\beta}\right) \\
& =f_{\beta}\left(f_{\alpha, \beta}\left(\boldsymbol{a}_{i}^{\alpha} \wedge_{\alpha} \boldsymbol{b}_{j}^{\alpha}\right) \vee\left(\boldsymbol{a}_{j}^{\beta} \wedge_{\beta} \boldsymbol{b}_{k}^{\beta}\right)\right) \\
& =f_{\beta}\left(\left(f_{\alpha, \beta}\left(\boldsymbol{a}_{i}^{\alpha} \wedge_{\alpha} \boldsymbol{b}_{j}^{\alpha}\right) \vee \boldsymbol{a}_{j}^{\beta}\right) \wedge_{\beta}\left(f_{\alpha, \beta}\left(\boldsymbol{a}_{i}^{\alpha} \wedge_{\alpha} \boldsymbol{b}_{j}^{\alpha}\right) \vee \boldsymbol{b}_{k}^{\beta}\right)\right) \\
& \geq f_{\beta}\left(f_{\gamma, \beta}\left(\boldsymbol{a}_{i}^{\gamma} \wedge_{\gamma} \boldsymbol{b}_{k}^{\gamma}\right)\right) \\
& =f_{\gamma}\left(\boldsymbol{a}_{i}^{\gamma} \wedge_{\gamma} \boldsymbol{b}_{k}^{\gamma}\right) \\
& =\boldsymbol{c}_{i, k},
\end{aligned}
$$

thus obtaining (2.5).

Case 2. $\beta \leq \alpha$. It follows from (2.4) that $\gamma \leq \alpha$ as well. In a fashion similar to Case 1, one can prove the following inequalities

$$
\begin{aligned}
& f_{\gamma, \alpha}\left(\boldsymbol{a}_{i}^{\gamma} \wedge_{\gamma} \boldsymbol{b}_{k}^{\gamma}\right) \leq \boldsymbol{b}_{j}^{\alpha} \vee f_{\beta, \alpha}\left(\boldsymbol{a}_{j}^{\beta} \wedge_{\beta} \boldsymbol{b}_{k}^{\beta}\right), \\
& f_{\gamma, \alpha}\left(\boldsymbol{a}_{i}^{\gamma} \wedge_{\gamma} \boldsymbol{b}_{k}^{\gamma}\right) \leq \boldsymbol{a}_{i}^{\alpha} \vee f_{\beta, \alpha}\left(\boldsymbol{a}_{j}^{\beta} \wedge_{\beta} \boldsymbol{b}_{k}^{\beta}\right),
\end{aligned}
$$

thus, as before, obtaining (2.5).

We summarize in Table 1 many known results and questions about uniform refinement properties, sometimes anticipating some subsequent sections of the present paper. We use the following abbreviations:

- distr. image of $\mathrm{gBs}=$ distributive image of a generalized Boolean semilattice;

- $L$ p.c. $=L$ with permutable congruences;

- $L$ s.c. $=L$ sectionally complemented;

- $L$ a.p.c. $=L$ with almost permutable congruences;

- $L$ r.c. $=L$ relatively complemented;

- $F_{\mathrm{b}}=\mathrm{FB}_{\mathbf{V}}\left(\omega_{2}\right)$, where $\mathbf{V}$ is a nondistributive variety of lattices;

- $F=\mathrm{F}_{\mathbf{V}}\left(\omega_{2}\right)$, where $\mathbf{V}$ is a nondistributive variety of lattices.

Also, the entry of the table marked by ${ }^{(*)}$ means that $\mathrm{URP}^{-}$holds in $\mathrm{Con}_{\mathrm{c}} L$ at principal congruences of $L$. Finally, we recall that $\mathrm{URP}^{+}$implies URP, which implies both WURP and $\mathrm{URP}^{-}$.

\begin{tabular}{|l|c|c|c|c|c|c|c|}
\cline { 2 - 8 } \multicolumn{1}{c|}{} & $\mathrm{Con}_{\mathrm{c}} F_{\mathrm{b}}$ & $\mathrm{Con}_{\mathrm{c}} F$ & $\begin{array}{l}\text { distr. image } \\
\text { of gBs }\end{array}$ & $\begin{array}{l}\mathrm{Con}_{\mathrm{c}} L, \\
L \text { p.c. }\end{array}$ & $\begin{array}{l}\mathrm{Con}_{\mathrm{c}} L, \\
L \text { s.c. }\end{array}$ & $\begin{array}{l}\mathrm{Con}_{\mathrm{c}} L, \\
L \text { a.p.c. }\end{array}$ & $\begin{array}{l}\text { Con } \\
L \text { r.c. }\end{array}$ \\
\hline $\mathrm{URP}^{+}$ & No & No & $?$ & $?$ & $?$ & $?$ & Yes \\
\hline $\mathrm{URP}$ & No & No & Yes & Yes & Yes & $?$ & Yes \\
\hline WURP & No & No & Yes & Yes & Yes & $?$ & Yes \\
\hline $\mathrm{URP}^{-}$ & No & No & Yes & Yes & Yes & Yes $^{(*)}$ & Yes \\
\hline
\end{tabular}

TABLE 1. Uniform refinement properties and congruence semilattices. 


\section{The $M_{3}\langle L\rangle$ construction, tensor product, and box product}

For a lattice $L$, we define $M_{3}\langle L\rangle$ as the set of all triples $\langle x, y, z\rangle \in L^{3}$ that are Boolean, that is, the following equalities hold:

$$
\begin{aligned}
& x=(x \vee y) \wedge(x \vee z), \\
& y=(y \vee x) \wedge(y \vee z), \\
& z=(z \vee x) \wedge(z \vee y) .
\end{aligned}
$$

The set $M_{3}\langle L\rangle$ is endowed with the restriction of the componentwise ordering on $L^{3}$. It can be shown that $M_{3}\langle L\rangle$ is a closure system in $L^{3}$, thus it is a lattice. Some of the relevant information about this construction is summarized in the following result, see G. Grätzer and F. Wehrung [25].

Proposition 3.1. For any lattice $L$, the following statements hold:

(i) For a congruence $\boldsymbol{a}$ of $L$, let $\boldsymbol{a}^{3}$ denote the congruence of $L^{3}$ defined as $\boldsymbol{a}$ componentwise. Let $M_{3}\langle\boldsymbol{a}\rangle$ be the restriction of $\boldsymbol{a}^{3}$ to $M_{3}\langle L\rangle$. Then $M_{3}\langle\boldsymbol{a}\rangle$ is a congruence of $M_{3}\langle L\rangle$, and every congruence of $M_{3}\langle L\rangle$ is of the form $M_{3}\langle\boldsymbol{a}\rangle$, for a unique congruence $\boldsymbol{a}$ of $L$.

(ii) The diagonal map $x \mapsto\langle x, x, x\rangle$ is a congruence-preserving embedding from $L$ into $M_{3}\langle L\rangle$. If, in addition, $L$ has a zero (resp., a unit), then the map $x \mapsto\langle x, 0,0\rangle$ (resp., $x \mapsto\langle 1, x, x\rangle$ ) is a congruence-preserving embedding from $L$ into $M_{3}\langle L\rangle$ whose range is an ideal (resp., a dual ideal) of $L$.

This solves the question raised above, namely, if $L$ is a nontrivial lattice, then the diagonal map from $L$ into $M_{3}\langle L\rangle$ defines a proper congruence-preserving extension of $L$.

We say that a lattice $L$ is regular, if any two congruences of $L$ that share a congruence class are equal. By iterating the $M_{3}\langle L\rangle$ construction, its refinement $M_{3}\langle L, a\rangle$ (the latter is a convex sublattice of $M_{3}\langle L\rangle$ ), and the gluing construction, G. Grätzer and E. T. Schmidt prove in [16] the following theorem:

Theorem 3.2. Every lattice $L$ has a congruence-preserving embedding into a regular lattice $\tilde{L}$. If $L$ has a zero, then one can suppose that $\tilde{L}$ has a zero and that $0_{L}=0_{\tilde{L}}$.

It is also observed in the same paper that every compact congruence of a regular lattice is principal. Hence, if CLP can be solved positively, then it can be solved with lattices in which every compact congruence is principal.

The $M_{3}\langle L\rangle$ construction has a far reaching generalization, the box product of lattices. The box product improves the classical tensor product of join-semilattices with zero, see, for example, G. Grätzer, H. Lakser, and R. W. Quackenbush [23] and G. Grätzer and F. Wehrung [28]. For $\langle\vee, 0\rangle$-semilattices $A$ and $B$, the tensor product $A \otimes B$ is defined in a fashion similar to the tensor product of vector spaces in linear algebra, in particular, it is also a $\langle\vee, 0\rangle$-semilattice. However, even in case both $A$ and $B$ are lattices, $A \otimes B$ is not necessarily a lattice, see G. Grätzer and F. Wehrung [26, 27]. 
For a lattice $L$, we put $\perp_{L}=\{0\}$, if $L$ has a zero (least element) 0 , and $\perp_{L}=\varnothing$, otherwise. For lattices $A$ and $B$ and $\langle a, b\rangle \in A \times B$, we define

$$
\begin{aligned}
& \perp_{A, B}=\left(A \times \perp_{B}\right) \cup\left(\perp_{A} \times B\right), \\
& a \otimes b=\perp_{A, B} \cup\{\langle x, y\rangle \in A \times B \mid x \leq a \text { and } y \leq b\}, \\
& a \square b=\{\langle x, y\rangle \in A \times B \mid x \leq a \text { or } y \leq b\} .
\end{aligned}
$$

We denote by $A \square B$ the box product of $A$ and $B$; the elements of $A \square B$ are subsets of $A \times B$ that can be represented as finite intersections of sets of the form $a \square b$, with $a \in A$ and $b \in B$. Unlike the tensor product $A \otimes B$, it is always a lattice, see G. Grätzer and F. Wehrung [29]. An element of $A \square B$ is confined, if it is contained in some element of the form $a \bigotimes b$, for $\langle a, b\rangle \in A \times B$. The ideal $A \otimes B$ of all confined elements of $A \square B$ is nonempty iff either $A$ or $B$ is bounded, or both $A$ and $B$ have a zero, or both $A$ and $B$ have a unit; we call it the lattice tensor product of $A$ and $B$, and then the Isomorphism Theorem holds, which implies the following formula:

$$
\left(\operatorname{Con}_{\mathrm{c}} A\right) \otimes\left(\operatorname{Con}_{\mathrm{c}} B\right) \cong \operatorname{Con}_{\mathrm{c}}(A \otimes B) .
$$

In particular, for a lattice $L$, the lattice tensor product $M_{3} \otimes L$ is isomorphic to the lattice $M_{3}\langle L\rangle$ introduced at the beginning of Section 3. Such an isomorphism is called a coordinatization, and sometimes provides a more convenient way to compute in lattice tensor products. Arbitrary lattice tensor products of bounded lattices are coordinatized in G. Grätzer and M. Greenberg [18, 19, 20, 21].

To prove the Isomorphism Theorem, one first needs to verify a more general result that extends the formula (3.1) to so-called capped sub-tensor products of $A$ and $B$, this is the main result of G. Grätzer and F. Wehrung [28]. Then, one needs to verify that if $A$ and $B$ are lattices with zero, then $A \otimes B$ is a capped sub-tensor product of $A$ and $B$, see G. Grätzer and F. Wehrung [29]. The isomorphism of (3.1) carries $\Theta_{A}\left(a, a^{\prime}\right) \otimes \Theta_{B}\left(b, b^{\prime}\right)$ to $\Theta_{A \otimes B}\left(\left(a \otimes b^{\prime}\right) \vee\left(a^{\prime} \otimes b\right), a^{\prime} \otimes b^{\prime}\right)$, for all $a \leq a^{\prime}$ in $A$ and $b \leq b^{\prime}$ in $B$.

This has an application to the following problem. We say that a lattice $L$ is an automorphism-preserving extension of a sublattice $K$, if every automorphism of $K$ extends to a unique automorphism of $L$ and $K$ is closed under all automorphisms of $L$. By iterating the box product construction together with gluing, G. Grätzer and F. Wehrung solve in [30] a problem already proposed in the first edition of the monograph G. Grätzer [9], by proving the following:

Theorem 3.3 (The Strong Independence Theorem for arbitrary lattices). For every nontrivial lattice $L_{\mathrm{C}}$ and every lattice $L_{\mathrm{A}}$, there exists a lattice $L$ that is both a congruence-preserving extension of $L_{\mathrm{C}}$ and an automorphism-preserving extension of $L_{\mathrm{A}}$. Furthermore, if both $L_{\mathrm{C}}$ and $L_{\mathrm{A}}$ have a zero, then $L$ can be taken a zeropreserving extension of both $L_{\mathrm{C}}$ and $L_{\mathrm{A}}$.

Because of the well-known result of G. Birkhoff that states that every group appears as the automorphism group of some lattice, it follows that for every nontrivial lattice $K$ and every group $G$, there exists a congruence-preserving extension $L$ of $K$ such that Aut $L \cong G$. Observe that $\operatorname{Con} K \cong \operatorname{Con} L$. 
The essential difficulty of the proof of Theorem 3.3 lies in the construction, for a given lattice $L_{\mathrm{C}}$, of a rigid, congruence-preserving extension $\bar{L}_{\mathrm{C}}$ of $L_{\mathrm{C}}$. This construction is performed in several steps. For a bounded lattice $L$, we denote by $M_{3}\lfloor L\rfloor$ the set of all triples $\langle x, y, z\rangle$ of $M_{3}\langle L\rangle$ such that either $x=0$ or $x=1$, partially ordered under inclusion. Then $M_{3}\lfloor L\rfloor$ is a lattice, and its congruence lattice is isomorphic to the lattice of all congruences of $L$ that are either coarse or for which the congruence class of zero is zero.

Moreover, by using the lattice tensor product, one associates, with every lattice $L_{\mathrm{C}}$, a "large enough" simple, bounded lattice $S$ (whose cardinality may be larger than that of $L_{\mathrm{C}}$ ). Put $T=M_{3}\lfloor S\rfloor$. For a principal dual ideal $J$ of $L_{\mathrm{C}}$, we glue $L_{\mathrm{C}}$, with the dual ideal $J$, with $V=T \otimes J$, with the ideal $p \otimes J$, where $p$ denotes the unique atom of $T$. The result of this construction is a congruence-preserving extension of $L_{\mathrm{C}}$. By iterating this construction transfinitely many times, we obtain a rigid, congruence preserving-extension $\bar{L}_{\mathrm{C}}$ of $L_{\mathrm{C}}$. Observe that the cardinality of $\bar{L}_{\mathrm{C}}$ may be larger than the cardinality of $L_{\mathrm{C}}$. Furthermore, the extension $\bar{L}_{\mathrm{C}}$ thus constructed has a strong indecomposability property called steepness.

By using much more elementary techniques introduced earlier in G. Grätzer and E. T. Schmidt [13], for every lattice $L_{\mathrm{A}}$, one can construct a simple, automorphismpreserving extension $\bar{L}_{\mathrm{A}}$ of $L_{\mathrm{A}}$. Now, if $L_{\mathrm{A}}$ and $L_{\mathrm{C}}$ are given, the extensions $\bar{L}_{\mathrm{A}}$ and $\bar{L}_{\mathrm{C}}$ are constructed, then we put $L=\bar{L}_{\mathrm{A}} \otimes \bar{L}_{\mathrm{C}}$. Since $\bar{L}_{\mathrm{A}}$ is simple, $L$ is a congruencepreserving extension of $\bar{L}_{\mathrm{C}}$, thus of $L_{\mathrm{C}}$. Furthermore, every automorphism of $L_{\mathrm{A}}$ induces an automorphism of $\bar{L}_{\mathrm{A}}$, thus an automorphism of $L$. By using the steepness of $\bar{L}_{\mathrm{C}}$, one can prove, and this is the hardest part of the proof, that there are no other automorphisms of $L$. Therefore, $L$ is an automorphism-preserving extension of $\bar{L}_{\mathrm{A}}$.

If $X$ and $Y$ are subsets of a lattice $L$, we say that a map $\varphi: X \rightarrow Y$ is algebraic, if there exists a lattice polynomial $\mathbf{p}$, with one variable and with parameters from $L$, such that $\varphi(x)=\mathbf{p}(x)$, for all $x \in X$. In G. Grätzer and E. T. Schmidt [17], the following result is established:

Theorem 3.4. Let $K$ be a bounded lattice, let $[a, b]$ and $[c, d]$ be intervals of $K$, and let $\varphi:[a, b] \rightarrow[c, d]$ be an isomorphism between these two intervals. Then $K$ has $a\left\langle\vee, \wedge, \varphi, \varphi^{-1}\right\rangle$-congruence-preserving extension into a bounded lattice $L$ such that both $\varphi$ and $\varphi^{-1}$ are algebraic in $L$, and $K$ is a convex sublattice of $L$. In particular, the congruence lattice of the partial algebra $\left\langle K, \vee, \wedge, \varphi, \varphi^{-1}\right\rangle$ is isomorphic to the congruence lattice of the bounded lattice $\langle L, \vee, \wedge\rangle$.

The construction of Theorem 3.4 uses refinements of the $M_{3}\langle L\rangle$ construction together with the box product construction and gluing. Furthermore, it does not require transfinite induction, in particular, it preserves finiteness. Starting with a relatively complemented lattice $K$, the extension $L$ constructed by Theorem 3.4 is not relatively complemented. Compare with Corollary 6.7.

This result is extended to a family of isomorphisms in G. Grätzer and E. T. Schmidt [17], and to a family of surjective homomorphisms between intervals without requiring the inverses (that is, $\varphi^{-1}$ ) in the extended language, see G. Grätzer, 
M. Greenberg, and E. T. Schmidt [22]. The latter construction involves the consideration of the lattice tensor product of the original lattice not with $M_{3}$, but with $N_{6}$, the six element sectionally complemented lattice obtained by replacing one of the lower prime intervals of the square by a square. The manipulation of the elements of the box product is made more convenient by the coordinatization of lattice tensor products studied in G. Grätzer and M. Greenberg [18, 19, 20, 21].

However, these methods alone are not sufficient to solve CLP, because of the following observation. They extend a lattice $K$ to a lattice $L$ whose congruence lattice is isomorphic to the lattice $\operatorname{Con}^{\Phi} K$ of all lattice congruences of $K$ having the substitution property with respect to all the operations of $\Phi$, where $\Phi$ is a set of partial unary functions on $K$. In particular, Con $L$ is isomorphic to an algebraic subset of Con $K$ (a subset $X$ of a lattice $A$ is algebraic, if it is closed under arbitrary meets and nonempty directed joins of $A$ ), hence $\operatorname{Con}_{\mathrm{c}} L$ is the image of $\mathrm{Con}_{\mathrm{c}} K$ under a weakly distributive $\langle\vee, 0\rangle$-homomorphism (namely, the one that with a compact congruence $\boldsymbol{a}$ associates the $\Phi$-congruence generated by $\boldsymbol{a}$ ), see Section 2. In particular, if $K$ is already obtained from an already known representation theorem, then $\mathrm{Con}_{\mathrm{c}} K$ satisfies the axiom URP (see Corollary 6.7 and the comments that follow it), thus, by Proposition 2.5(ii), $\mathrm{Con}_{\mathrm{c}} L$ also satisfies URP.

Still this does not rule out the following possible approach of CLP, hinted at in Problem 1 in G. Grätzer and E. T. Schmidt [17]. If one could prove that every algebraic distributive lattice is isomorphic to some lattice of the form $\operatorname{Con}^{\Phi} K$, where $K$ is a lattice with zero and $\Phi$ is a set of partial surjective homomorphisms between intervals of $K$ satisfying certain simple conditions, then CLP would be solved positively. Observe that this would imply that every algebraic distributive lattice $D$ is isomorphic to an algebraic subset of some algebraic lattice of the form Con $K$; hence, if the semilattice of compact elements of $D$ does not satisfy URP, then neither does the semilattice $\mathrm{Con}_{\mathrm{c}} K$. Thus a natural guess would be to start with $K$ being, say, a free lattice.

The Isomorphism Theorem (see (3.1)) has another interesting consequence, see G. Grätzer and F. Wehrung [29]. We say that a $\langle\vee, 0\rangle$-semilattice $S$ is $\langle 0\rangle$-representable (resp., $\langle 0,1\rangle$-representable), if there exists a lattice $L$ with zero (resp., a bounded lattice $L$ ) such that $S \cong \operatorname{Con}_{\mathrm{c}} L$. The problem whether any representable semilattice $S$ (i.e., a semilattice $S$ for which there exists a lattice $L$ such that $\left.S \cong \operatorname{Con}_{\mathrm{c}} L\right)$ is $\langle 0\rangle$-representable is open.

Theorem 3.5. Let $S$ and $T$ be $\langle\vee, 0\rangle$-semilattices. Then the following statements hold:

(i) If both $S$ and $T$ are $\langle 0\rangle$-representable, then $S \otimes T$ is $\langle 0\rangle$-representable.

(ii) If both $S$ and $T$ are $\langle 0,1\rangle$-representable, then $S \otimes T$ is $\langle 0,1\rangle$-representable.

(iii) If $S$ is representable and $T$ is $\langle 0,1\rangle$-representable, then $S \otimes T$ is representable.

This result can be easily extended to iterated tensor products of $\langle\vee, 0,1\rangle$-semilattices. For $\langle\vee, 0,1\rangle$-semilattices $S$ and $T$, the rule $x \mapsto x \otimes 1_{T}$ defines a $\langle\vee, 0,1\rangle$ embedding from $S$ into $S \otimes T$. For a family $\left\langle S_{i} \mid i \in I\right\rangle$ of $\langle\vee, 0,1\rangle$-semilattices and finite subsets $I_{0} \subseteq I_{1}$ of $I$, one defines similarly a $\langle\vee, 0,1\rangle$-embedding from $\bigotimes_{i \in I_{0}} S_{i}$ 
into $\bigotimes_{i \in I_{1}} S_{i}$. These maps obviously form a direct system of $\langle\vee, 0,1\rangle$-semilattices and $\langle\vee, 0,1\rangle$-embeddings; let $\bigotimes_{i \in I} S_{i}$ denote its direct limit, the iterated tensor product of the $S_{i}$-s. Suppose now that $S_{i}=\operatorname{Con}_{\mathrm{c}} L_{i}$, for some bounded lattice $L_{i}$, for all $i \in I$. By arguing as for semilattices except that $\otimes$ is replaced by $\otimes$, we obtain a $\langle 0,1\rangle$-lattice embedding from $\bigotimes_{i \in I_{0}} L_{i}$ into $\bigotimes_{i \in I_{1}} L_{i}$. These maps also form a direct system; denote its direct limit by $\bigotimes_{i \in I} L_{i}$. Since the $\operatorname{Con}_{\mathrm{c}}$ functor preserves direct limits (see Proposition 5.1), we obtain the formula

$$
\operatorname{Con}_{\mathrm{c}}\left(\bigotimes_{i \in I} L_{i}\right) \cong \bigotimes_{i \in I}\left(\operatorname{Con}_{\mathrm{c}} L_{i}\right)
$$

This yields the following result:

Theorem 3.6. Any iterated tensor product of $\langle 0,1\rangle$-representable $\langle\vee, 0\rangle$-semilattices is $\langle 0,1\rangle$-representable.

This result is similar to K. R. Goodearl and D. E. Handelman [7, Theorem 3.5], where it is proved that for any family $\left\langle G_{i} \mid i \in I\right\rangle$ of dimension groups with orderunit, if every $G_{i}$ is isomorphic to the $K_{0}$ of some locally matricial algebra (over a given field), then so is the iterated tensor product $\bigotimes_{i \in I} G_{i}$.

\section{The functor $\mathrm{Con}_{\mathrm{c}}$ on partial lattices}

Throughout the paper we shall make use of the following categories:

- $\mathbf{L}$, the category of all lattices and lattice homomorphisms;

- $\mathbf{S}$, the category of all $\langle\vee, 0\rangle$-semilattices and $\langle\vee, 0\rangle$-homomorphisms;

- $\mathbf{S}_{\mathbf{d}}$, the full subcategory of $\mathbf{S}$ whose objects are the distributive $\langle\vee, 0\rangle$-semilattices;

- $\mathbf{S}_{\mathbf{f d}}$, the full subcategory of $\mathbf{S}$ whose objects are the finite distributive $\langle\vee, 0\rangle$-semilattices;

- $\mathbf{S}_{\mathbf{f b}}$, the full subcategory of $\mathbf{S}$ whose objects are the finite Boolean $\langle\vee, 0\rangle$ semilattices.

The correspondence that with every lattice $L$ associates its congruence semilattice $\operatorname{Con}_{\mathrm{c}} L$ can be extended to a functor from $\mathbf{L}$ to $\mathbf{S}$. For a lattice homomorphism $f: K \rightarrow L$, let $\operatorname{Con}_{\mathrm{c}} f$ be the map from $\operatorname{Con}_{\mathrm{c}} K$ to $\operatorname{Con}_{\mathrm{c}} L$ that with every compact congruence $\boldsymbol{a}$ associates the congruence of $L$ generated by all pairs $\langle f(x), f(y)\rangle$, where $\langle x, y\rangle \in \boldsymbol{a}$.

This can be easily extended to partial lattices. The precise concepts are summarized in the following two definitions (see F. Wehrung [52]). We observe that the definition of a partial lattice that we use here is very closely related to the one used in R. Freese, J. Ježek, and J. B. Nation [4] but not to the one in G. Grätzer [9].

\section{Definition 4.1.}

(i) A partial prelattice is a structure $\langle P, \leq, \bigvee, \wedge\rangle$, where $P$ is a nonempty set, $\leq$ is a quasi-ordering on $P$, and $\bigvee, \wedge$ are partial functions from the set $[P]_{*}^{<\omega}$ of all nonempty finite subsets of $P$ to $P$ satisfying the following properties: 
(a) $a=\bigvee X$ implies that $a=\sup X$, for all $a \in P$ and all $X \in[P]_{*}^{<\omega}$.

(b) $a=\bigwedge X$ implies that $a=\inf X$, for all $a \in P$ and all $X \in[P]_{*}^{<\omega}$.

(By $a=\sup X$, we mean that an element $b$ of $P$ is an upper bound of $X$ iff $a \leq b$. The statement $a=\inf X$ is defined dually.)

(ii) $P$ is a partial lattice, if $\leq$ is antisymmetric.

(iii) A congruence of $P$ is a quasi-ordering $\preceq$ of $P$ containing $\leq$ such that $\langle P, \preceq, \bigvee, \wedge\rangle$ is a partial prelattice.

Lattices are naturally identified with partial lattices $P$ such that $\bigvee$ and $\Lambda$ are defined for all finite subsets of $P$. We denote by $\operatorname{Con}_{\mathrm{c}} P$ the $\langle\mathrm{V}, 0\rangle$-semilattice of all compact congruences of $P$. The compact congruences of $P$ are those of the form

$$
\bigvee_{i<n} \Theta^{+}\left(a_{i}, b_{i}\right)
$$

where $n<\omega, a_{0}, \ldots, a_{n-1}, b_{0}, \ldots, b_{n-1} \in P$, and where we define $\Theta^{+}\left(a_{i}, b_{i}\right)$ to be the least congruence $\preceq$ of $P$ such that $a_{i} \preceq b_{i}$. We observe that congruences of a partial lattice $P$ are no longer equivalence relations on $P$ but quasi-orderings of $P$.

Definition 4.2. If $P$ and $Q$ are partial prelattices, a homomorphism of partial prelattices from $P$ to $Q$ is an order-preserving map $f: P \rightarrow Q$ such that $a=\bigvee X$ (resp., $a=\bigwedge X$ ) implies that $f(a)=\bigvee f[X]$ (resp., $f(a)=\bigwedge f[X]$ ), for all $a \in P$ and all $X \in[P]_{*}^{<\omega}$. We say that a homomorphism $f$ is an embedding, if $f(a) \leq f(b)$ implies that $a \leq b$, for all $a, b \in P$.

For a homomorphism $f: P \rightarrow Q$ of partial lattices the map $\operatorname{Con}_{\mathrm{c}} f: \operatorname{Con}_{\mathrm{c}} P \rightarrow$ $\mathrm{Con}_{\mathrm{c}} Q$ assigns to every compact congruence $\boldsymbol{a}$ of $P$ the congruence of $Q$ generated by all the pairs $\langle f(x), f(y)\rangle$, for $\langle x, y\rangle \in \boldsymbol{a}$. This way, the correspondence $\mathrm{Con}_{\mathrm{c}}$ becomes a functor from the category PL of partial lattices and homomorphisms of partial lattices to the category $\mathbf{S}$.

\section{Lifting diagrams of semilattices by diagrams of partial lattices}

In this section, we shall review some useful categorical concepts.

Every poset $I$ can be viewed as a category, whose set of objects is $I$, where for all $p, q \in I$, there exists a morphism from $p$ to $q$ exactly when $p \leq q$, and then this morphism is unique; we shall denote it by $p \rightarrow q$. We shall often identify a poset with its associated category.

For a category $\mathbf{C}$, we shall say that a diagram of $\mathbf{C}$ is a functor $\mathcal{D}: I \rightarrow \mathbf{C}$, where $I$ is a poset. If $\mathbf{D}$ is another category, a functor $\Phi: \mathbf{C} \rightarrow \mathbf{D}$ is said to preserve direct limits ( $=$ directed colimits), if whenever $\mathcal{D}$ is a diagram of $\mathbf{C}$ indexed by a directed poset $I$ and $X=\lim \mathcal{D}$ in $\mathbf{C}$, then $\Phi(X)=\lim (\Phi \mathcal{D})$. Observe that this standard category-theoretical formulation abuses notation in two ways:

- Strictly speaking, $X$ does not simply consist of an object of $\mathbf{C}$ but rather of an object of $\mathbf{C}$ together with a family of morphisms $\mathcal{D}(i) \rightarrow X$, for $i \in I$, satisfying natural commutation relations.

- The statement $X=\underline{\lim } \mathcal{D}$ determines $X$ only up to isomorphism. 
Proposition 5.1. The functor $\mathrm{Con}_{\mathrm{c}}$ from partial lattices to $\langle\vee, 0\rangle$-semilattices preserves direct limits.

Of course, Proposition 5.1 is not specific to partial lattices, it is an easy basic fact of universal algebra that holds for any "reasonable" definition of a partial algebra.

Now let us see how this can help us tackle CLP.

Since every distributive semilattice $D$ is the direct union of its finite distributive subsemilattices (see P. Pudlák [39]), one can start with a diagram $\mathcal{E}: I \rightarrow \mathbf{S}_{\mathbf{f d}}$ indexed by a directed poset $I$ and try to find a diagram $\mathcal{D}: I \rightarrow \mathbf{L}$ such that $\lim _{\mathcal{E}} \operatorname{Con}_{\mathrm{c}} \mathcal{D}$ and $\lim _{\mathcal{E}} \mathcal{E}$ are isomorphic. This is true if the functors $\operatorname{Con}_{\mathrm{c}} \mathcal{D}$ and $\overrightarrow{\mathcal{E}}: I \rightarrow \mathbf{S}_{\mathbf{f d}}$ are naturally equivalent (see P. Pudlák [39]), that is, if there exists a system $\left\langle\varepsilon_{i} \mid i \in I\right\rangle$ of isomorphisms $\varepsilon_{i}: \operatorname{Con}_{\mathrm{c}} \mathcal{D}(i) \rightarrow \mathcal{E}(i)$ (for $i \in I$ ) such that the diagram of Figure 2 commutes, for all $i \leq j$ in $I$. If this is the case, then we also

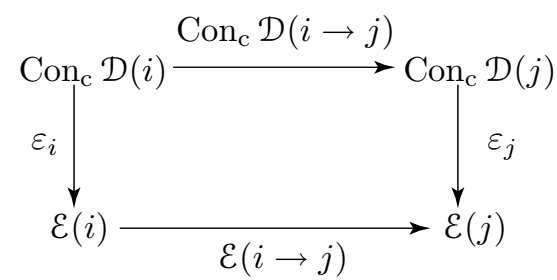

Figure 2. Natural equivalence of $\operatorname{Con}_{\mathrm{c}} \mathcal{D}$ and $\mathcal{E}$.

say that $\mathcal{D}$ lifts $\mathcal{E}$ with respect to $\mathrm{Con}_{\mathrm{c}}$.

In some cases, lifts of diagrams can be constructed inductively using the following concepts, see F. Wehrung [53]. By a morphism $\varphi: \mathcal{E} \rightarrow S$ of a diagram $\mathcal{E}: I \rightarrow \mathbf{S}$ to an object $S$ of $\mathbf{S}$ we mean a family $\left\langle\varphi_{i} \mid i \in I\right\rangle$ of morphisms $\varphi_{i}: \mathcal{E}(i) \rightarrow S$ of $\mathbf{S}$ such that the equality $\varphi_{i}=\varphi_{j} \circ \mathcal{E}(i \rightarrow j)$ holds, for all $i \leq j$ in $I$.

Definition 5.2. Let $\mathcal{D}$ be a diagram of partial lattices. For a $\langle\vee, 0\rangle$-semilattice $S$ and a partial lattice $P$, we say that a homomorphism $\varphi$ : $\operatorname{Con}_{\mathrm{c}} \mathcal{D} \rightarrow S$ can be

(i) factored through $P$, if there are a homomorphism $f: \mathcal{D} \rightarrow P$ and a $\langle\vee, 0\rangle$ homomorphism $\psi: \operatorname{Con}_{\mathrm{c}} P \rightarrow S$ such that $\varphi=\psi \circ \operatorname{Con}_{\mathrm{c}} f$

(ii) lifted through $P$, if there are a homomorphism $f: \mathcal{D} \rightarrow P$ and an isomorphism $\psi: \operatorname{Con}_{\mathrm{c}} P \rightarrow S$ such that $\varphi=\psi \circ \operatorname{Con}_{\mathrm{c}} f$.

In (i) (resp., (ii)) above, we say that $\varphi$ can be factored to (resp., lifted to) $f$.

For example, any distributive semilattice $C$ is isomorphic to the direct limit of a diagram $\mathcal{E}:[C \times \omega]^{<\omega} \rightarrow \mathbf{S}_{\mathbf{f b}}$ of finite Boolean semilattices, see K. R. Goodearl and $\mathrm{F}$. Wehrung [8] $\left([X]^{<\omega}\right.$ denotes the set of all finite subsets of a set $\left.X\right)$. So if this diagram could be lifted with respect to the functor $\mathrm{Con}_{\mathrm{c}}$, then it would give a proof that every distributive semilattice is isomorphic to the semilattice of compact congruences of a lattice.

By a truncated $n$-cube of lattices, we mean a functor $\mathcal{E}: \mathcal{P}_{<}(n) \rightarrow \mathbf{L}$, where we put $\mathcal{P}_{<}(n)=\mathcal{P}(n) \backslash\{n\}$, partially ordered by inclusion. An inductive construction 
of a lift (with respect to $\mathrm{Con}_{\mathrm{c}}$ ) of a diagram $\varepsilon:[C \times \omega]^{<\omega} \rightarrow \mathbf{S}_{\mathrm{fb}}$ then requires constructing lifts of homomorphisms of the form $\varphi_{n}: \operatorname{Con}_{\mathrm{c}} \mathcal{E}_{n} \rightarrow D$, where $n$ is a natural number, $D$ is a distributive semilattice, and $\mathcal{E}_{n}: \mathcal{P}_{<}(n) \rightarrow \mathbf{L}$ is a truncated $n$-cube of lattices, through a lattice $L$. This suggests that the study of the following $n$-dimensional versions of CLP may be of interest.

Definition 5.3. For a natural number $n$, we say that a $\langle\vee, 0\rangle$-semilattice $S$ satisfies $n$-dimensional CLP, or $n$-CLP in short, if for every truncated $n$-cube $\mathcal{E}$ of lattices, every homomorphism $\varphi: \operatorname{Con}_{\mathrm{c}} \mathcal{E} \rightarrow S$ can be lifted.

Thus, $(n+1)$-CLP at $S$ is stronger than $n$-CLP at $S$, while 0-CLP at $S$ is equivalent to the statement that $S \cong \operatorname{Con}_{\mathrm{c}} L$, for some lattice $L$. It is worthwhile to restate $n$-CLP at $S$, for $n \in\{1,2\}$ :

1-CLP. The property 1-CLP holds at $S$ iff for every lattice $K$, every $\langle\vee, 0\rangle$-homomorphism $\varphi: \operatorname{Con}_{\mathrm{c}} K \rightarrow S$ can be lifted, that is, there are a lattice $L$, a lattice homomorphism $f: K \rightarrow L$, and an isomorphism $\varepsilon: \operatorname{Con}_{\mathrm{c}} L \rightarrow S$ such that $\varphi=\varepsilon \circ \operatorname{Con}_{\mathrm{c}} f$, as illustrated on Figure 3 .

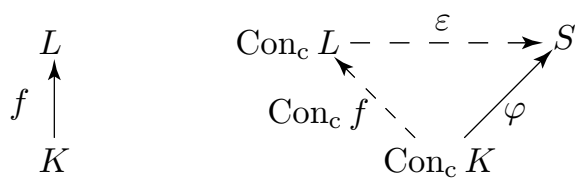

FigURE 3. Illustrating 1-CLP at $S$.

2-CLP. The property 2-CLP holds at $S$ iff for all lattices $K_{0}, K_{1}, K_{2}$, all lattice homomorphisms $f_{i}: K_{0} \rightarrow K_{i}$ and all $\langle\vee, 0\rangle$-homomorphisms $\varphi_{i}: \operatorname{Con}_{\mathrm{c}} K_{i} \rightarrow$ $S$, for $i \in\{1,2\}$ such that $\varphi_{1} \circ \operatorname{Con}_{\mathrm{c}} f_{1}=\varphi_{2} \circ \operatorname{Con}_{\mathrm{c}} f_{2}$, there are a lattice $L$, lattice homomorphisms $g_{i}: K_{i} \rightarrow L$, for $i \in\{1,2\}$, and an isomorphism $\varepsilon: \operatorname{Con}_{\mathrm{c}} L \rightarrow S$ such that $g_{1} \circ f_{1}=g_{2} \circ f_{2}$ and $\varepsilon \circ \operatorname{Con}_{\mathrm{c}} g_{i}=\varphi_{i}$, for $i \in\{1,2\}$, as illustrated on Figure 4 .

For $n \geq 3$, it follows from a simple example in F. Wehrung [53] that $n$-CLP has a trivial answer:

Proposition 5.4. Let $S$ be $a\langle\vee, 0\rangle$-semilattice. Then 3-CLP holds at $S$ iff $S=\{0\}$.

A much harder related result is proved in J. Tưma and F. Wehrung [45]:

Theorem 5.5. There exists a 3 -cube $\mathcal{E}: \mathcal{P}(3) \rightarrow \mathbf{S}_{\mathbf{f b}}$ that has no lift $\mathcal{D}: \mathcal{P}(3) \rightarrow \mathbf{L}$ with respect to $\mathrm{Con}_{\mathrm{c}}$ such that $\mathcal{D}(\{i\})$ has (almost) permutable congruences, for all $i<3$.

Here, we say that a lattice $L$ has permutable congruences (resp., almost permutable congruences), if $\boldsymbol{a} \boldsymbol{b}=\boldsymbol{b} \boldsymbol{a}$ (resp., $\boldsymbol{a} \vee \boldsymbol{b}=\boldsymbol{a} \boldsymbol{b} \cup \boldsymbol{b} \boldsymbol{a}$ ), for all congruences $\boldsymbol{a}$ and $\boldsymbol{b}$ of $L$. The basic idea underlying the proof of Theorem 5.5 consists of extracting the combinatorial core of Theorem 2.8. 

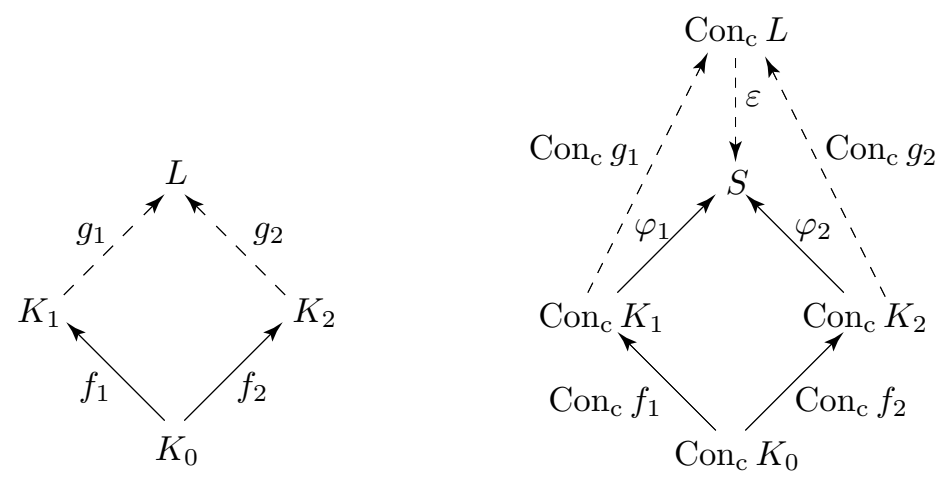

Figure 4. Illustrating 2-CLP at $S$.

\section{Extensions of partial lattices to lattices}

The main result of F. Wehrung [52], together with the converse proved in J. Tưma and F. Wehrung [46], imply the following.

Theorem 6.1. Let $S$ be a distributive $\langle\vee, 0\rangle$-semilattice $S$. Then 1-CLP holds at $S$ iff $S$ is a lattice.

The proof that 1-CLP at $S$ implies that $S$ is a lattice is established in J. Tưma and F. Wehrung [46], and it uses an ad hoc construction: for a distributive $\langle\vee, 0\rangle$ semilattice $S$ that is not a lattice, one constructs a Boolean lattice $B$ of cardinality $2^{|S|}$ and a $\langle\vee, 0\rangle$-homomorphism $\varphi: \operatorname{Con}_{\mathrm{c}} B \rightarrow S$ without a lift.

The proof that $S$ being a lattice implies 1-CLP at $S$ is very long and difficult, although the basic idea is quite simple. We are given a distributive $\langle\vee, 0\rangle$-semilattice $S$, a lattice $K$, and a $\langle\vee, 0\rangle$-homomorphism $\varphi: \operatorname{Con}_{\mathrm{c}} K \rightarrow S$. This information can be conveniently expressed by saying that the pair $\langle K, \varphi\rangle$ is a $S$-measured lattice. We extend $\langle K, \varphi\rangle$ to a pair $\langle L, \psi\rangle$, by successive one-step extensions starting on $\langle K, \varphi\rangle$ that can be informally described as follows:

(i) Let $a<b<c$ in $K$. We freely adjoin to $K$ a relative complement $x$ of $b$ in the interval $[a, c]$. Then the map $\psi$ sends $\Theta_{L}(a, x)$ to $\varphi \Theta_{K}(b, c)$ and $\Theta_{L}(x, c)$ to $\varphi \Theta_{K}(a, b)$.

(ii) Let $\boldsymbol{a}$ and $\boldsymbol{b}$ be congruences of $K$ such that $\varphi(\boldsymbol{a})=\varphi(\boldsymbol{b})$. Then $L$ is constructed such that $\left(\operatorname{Con}_{\mathrm{c}} j\right)(\boldsymbol{a})=\left(\operatorname{Con}_{\mathrm{c}} j\right)(\boldsymbol{b})$, where $j: K \rightarrow L$ is a lattice homomorphism, while the construction is "sufficiently free".

(iii) Let $\alpha \in S$ be not in the range of $\varphi$, fix $o \in K$ and add freely to $K$ an outside element $x>o$. Let $\psi$ send $\Theta_{L}(o, x)$ to $\alpha$.

Iterating these steps transfinitely should, intuitively, yield an $S$-measured lattice $\langle L, \psi\rangle$ with $L$ relatively complemented and $\psi$ an isomorphism. This approach suffers from many drawbacks:

(a) The structure $L$ obtained above is not a lattice, but a partial lattice. Hence the induction step should be performed not on a pair $\langle K, \varphi\rangle$ with $K$ a 
lattice, but on a pair $\langle P, \varphi\rangle$, where $P$ is a partial lattice and $\varphi: \operatorname{Con}_{\mathrm{c}} P \rightarrow S$ is a $\langle\vee, 0\rangle$-homomorphism. We shall say that $\langle P, \varphi\rangle$ is a $S$-measured partial lattice.

(b) Performing the steps above on an $S$-measured partial lattice $\langle P, \varphi\rangle$ leads to the problem of extending the map $\varphi$ that may not have a solution even in simple cases.

(c) Once the inductive construction is completed, taking the limit does not yield a lattice, but a partial lattice.

Item (a) is taken care of by the extension of the $\mathrm{Con}_{\mathrm{c}}$ functor to the category $\mathbf{P L}$ of partial lattices, as presented in Section 4 .

Item (b) is much more difficult to take care of. In F. Wehrung [52], this is done by considering a certain class of $S$-measured partial lattices $\langle P, \varphi\rangle$ that are called balanced $S$-measured partial lattices, for which the extension from $\varphi$ to $\psi$ can be performed. The formal definition of being balanced is quite complicated. Intuitively, it means that the meet and join operations can be computed in the ideal and filter lattices of $P$ by focusing attention on finite sets of elements, called there samples, and do this uniformly on all quotients of $P$.

Item (c) is taken care in a similar fashion as item (b). For a partial lattice $P$, let $\mathrm{F}_{\mathbf{L}}(P)$ denote the free lattice on $P$, see R.P. Dilworth [3], R. A. Dean [2], or R. Freese, J. Ježek, and J. B. Nation [4]. Then the canonical map from $\operatorname{Con}_{\mathrm{c}} P$ to $\operatorname{Con}_{\mathrm{C}} \mathrm{F}_{\mathbf{L}}(P)$ is a cofinal $\langle\vee, 0\rangle$-embedding. This alone is not sufficient to ensure the existence of an extension $\psi: \operatorname{Con}_{\mathrm{C}} \mathrm{F}_{\mathbf{L}}(P) \rightarrow S$ of the map $\varphi: \operatorname{Con}_{\mathrm{c}} P \rightarrow S$, however, this is possible if $\langle P, \varphi\rangle$ is balanced. The formula defining the extension $\psi$ may be better understood by viewing $\langle P, \varphi\rangle$ as a $S^{\mathrm{d}}$-valued partial lattice, where $S^{\mathrm{d}}$ denotes the dual lattice of $S$ (thus it is a distributive lattice with 1 ). Thus we need to deal with structures similar to the Boolean-valued models encountered in forcing (e.g., in set theory), except that the "truth values" live not in a complete Boolean algebra but in the lattice $S^{\mathrm{d}}$.

Once all these problems are solved, we obtain the following much stronger result, see Theorem D in F. Wehrung [52]:

Theorem 6.2. Let $S$ be a distributive lattice with zero. Then for all lattices $K_{0}$, $K_{1}, K_{2}$ with $K_{0}$ finite, all lattice homomorphisms $f_{i}: K_{0} \rightarrow K_{i}$ and all $\langle\vee, 0\rangle$-homomorphisms $\varphi_{i}: \operatorname{Con}_{\mathrm{c}} K_{i} \rightarrow S$, for $i \in\{1,2\}$ such that $\varphi_{1} \circ \operatorname{Con}_{\mathrm{c}} f_{1}=\varphi_{2} \circ \operatorname{Con}_{\mathrm{c}} f_{2}$, there are a lattice $L$, lattice homomorphisms $g_{i}: K_{i} \rightarrow L$, for $i \in\{1,2\}$, and an isomorphism $\varepsilon: \operatorname{Con}_{\mathrm{c}} L \rightarrow S$ such that $\varepsilon \circ \operatorname{Con}_{\mathrm{c}} g_{i}=\varphi_{i}$, for $i \in\{1,2\}$. Furthermore, $L, g_{1}$, and $g_{2}$ can be found in such a way that the following statements hold:

(i) $L$ is relatively complemented.

(ii) $g_{1}\left[K_{1}\right] \cup g_{2}\left[K_{2}\right]$ generates $L$ as an ideal (resp., a filter).

(iii) If $\operatorname{rng} \varphi_{1} \cup \operatorname{rng} \varphi_{2}$ generates $S$ as an ideal, then $g_{1}\left[K_{1}\right] \cup g_{2}\left[K_{2}\right]$ generates $L$ as a convex sublattice.

This is a far reaching generalization of the original result of M. Tischendorf [43] with $K_{0}=K_{1}=K_{2}$ finite, $f_{1}=f_{2}=\mathrm{id}, S$ finite, and $\varphi$ an embedding.

The lifts satisfying conditions (i)-(iii) in Theorem 6.2 are called good lifts in F. Wehrung [52]. The properties of $\langle L, \varepsilon\rangle$ from which they follow are reminiscent 
of genericity (in the model-theoretical sense), and these properties have further consequences, for example, the lattice $L$ has definable congruence inclusion in the sense that for every positive integer $n$, there exists a positive existential formula (independent of the lattice $L) \Phi_{n}\left(\mathrm{x}_{0}, \mathrm{y}_{0}, \mathrm{x}_{1}, \mathrm{y}_{1}, \ldots, \mathrm{x}_{n}, \mathrm{y}_{n}\right)$ of lattice theory such that $L$ satisfies that

$$
\Theta\left(x_{0}, y_{0}\right) \subseteq \bigvee_{i=1}^{n} \Theta\left(x_{i}, y_{i}\right) \quad \text { iff } \quad L \text { satisfies } \Phi_{n}\left(x_{0}, y_{0}, x_{1}, y_{1}, \ldots, x_{n}, y_{n}\right),
$$

for any $x_{0}, y_{0}, \ldots, x_{n}, y_{n} \in L$. As for the lifts of truncated squares (the case $n=2$ ) earlier results by the first author, J. Tůma [44] and also G. Grätzer, H. Lakser, and F. Wehrung [10] are extended to infinite semilattices in F. Wehrung [53].

Definition 6.3. A $\langle\vee, 0\rangle$-semilattice $S$ is called conditionally co-Brouwerian, if the following holds:

(i) for all nonempty subsets $X$ and $Y$ of $S$ such that $X \leq Y$ (that is, $x \leq y$, for all $x \in X$ and $y \in Y$ ), there exists $z \in S$ such that $X \leq z \leq Y$ (we then say that $S$ is conditionally complete);

(ii) for every subset $Z$ of $S$, if $a \leq b \vee z$, for all $z \in Z$, then there exists $c \in S$ such that $a \leq b \vee c$ and $c \leq \bar{Z}$.

By restricting this definition to subsets $X, Y$, and $Z$ of $S$ such that $|X|,|Y|$, $|Z|<\kappa$, for an infinite cardinal $\kappa$, we define conditionally $\kappa$-co-Brouwerian $\langle\vee, 0\rangle$ semilattices.

Of course, any conditionally co-Brouwerian $\langle\vee, 0\rangle$-semilattice is a distributive lattice with zero.

Theorem 6.4. Let $\mathcal{E}$ be a truncated square of partial lattices with $\mathcal{E}(\varnothing)$ a lattice, let $S$ be a conditionally co-Brouwerian lattice. Then every homomorphism $\varphi: \operatorname{Con}_{\mathrm{c}} \mathcal{E} \rightarrow S$ has a good lift.

A self-contained proof of Theorem 6.4 is significantly easier than a self-contained proof of Theorem 6.2, because for a conditionally co-Brouwerian lattice $S$ and a partial lattice $P$, any $\langle\vee, 0\rangle$-homomorphism $\varphi: \operatorname{Con}_{\mathrm{c}} P \rightarrow S$ can be extended to a $\langle\vee, 0\rangle$-homomorphism $\psi: \operatorname{Con}_{\mathrm{C}} \mathrm{F}_{\mathbf{L}}(P) \rightarrow S$; this follows from monoid-theoretical considerations introduced in F. Wehrung [48]. The assumption that $S$ is conditionally co-Brouwerian implies that $S$ is injective in a suitable category of partially quasi-ordered monoids.

As a consequence of one- and two-dimensional lifting results in F. Wehrung $[52,53]$, we obtain the following extensions of known 0-dimensional results:

Theorem 6.5. Every member in each of the following classes of $\langle\vee, 0\rangle$-semilattices is isomorphic to $\mathrm{Con}_{\mathrm{c}} L$, for some relatively complemented lattice $L$ with zero that has definable congruence inclusion:

(i) $\langle\vee, 0\rangle$-direct limits of the form $\underline{\lim }_{n \in \omega} S_{n}$, where all the $S_{n}$ are distributive lattices with zero;

(ii) $\langle\vee, 0\rangle$-direct limits of the form $\lim _{i \in I} S_{i}$, where $I$ is a directed poset of cardinality at most $\aleph_{1}$ and all the $\vec{S}_{i}$, for $i \in I$, are conditionally co-Brouwerian; 
(iii) All $\langle\vee, 0\rangle$-semilattices $S$ that are conditionally $|S|$-co-Brouwerian.

Item (i) above extends the main result of E. T. Schmidt [42] (any distributive lattice with zero is representable), while (ii) above extends the main result of A.P. Huhn $[32,33]$ (any distributive $\langle\vee, 0\rangle$-semilattice of cardinality at most $\aleph_{1}$ is representable). Item (iii), first stated and proved in F. Wehrung [53], seems to be completely new.

We recall here the following result, obtained by E. T. Schmidt, see [41]:

Theorem 6.6 (Schmidt's Lemma). Let B be a generalized Boolean semilattice. Then any image of $B$ under a distributive homomorphism (see Definition 2.4) is isomorphic to $\operatorname{Con}_{\mathrm{c}} L$, for some lattice $L$.

We obtain the following informal corollary:

Corollary 6.7. All the representation theorems of distributive $\langle\vee, 0\rangle$-semilattices other than Schmidt's Lemma that are known to this point yield semilattices that are representable by relatively complemented lattices with zero and with definable congruence inclusion.

It is unclear whether every image $S$ of a generalized Boolean semilattice under a distributive homomorphism can be represented as $\operatorname{Con}_{c} L$, for a relatively complemented lattice $L$. However, a direct verification yields that $S$ satisfies the axiom URP considered in Section 2 (see Proposition 2.5(ii)), and the (easy) proof fails for the stronger axiom $\mathrm{URP}^{+}$(see Definition 2.9). On the other hand, the semilattice $\mathrm{Con}_{\mathrm{c}} L$ satisfies $\mathrm{URP}^{+}$, for any relatively complemented lattice $L$ (see Proposition 2.10); see also Problem 10.

In any case, all known representation theorems (Schmidt's Lemma included) yield semilattices that satisfy the axiom URP studied in Section 2. In particular, none of them is able to reach $\operatorname{Con}_{\mathbf{c}} \mathrm{F}_{\mathbf{L}}\left(\omega_{2}\right)$, although this semilattice is already represented!

\section{Connections to ring theory}

The paper K. R. Goodearl and F. Wehrung [8] is a rich source of information on connections between congruence lattice representation problems and ring theory. For our present purpose, we mention the following theorem that goes back to J. von Neumann [35]. A ring (associative, not necessarily with unit) $R$ is called regular (in von Neumann's sense), if for all $x \in R$, there exists $y \in R$ such that $x y x=x$. We recall the following classical result, see K. D. Fryer and I. Halperin [5] for a proof of the case without unit:

Proposition 7.1. The set $\mathcal{L}(R)$ of all principal right ideals of a regular ring $R$, ordered under inclusion, is a sectionally complemented modular lattice.

This together with the following result from F. Wehrung [50] gives a strategy for representing distributive semilattices as semilattices of compact congruences of sectionally complemented modular lattices. 
Proposition 7.2. Let $R$ be a regular ring. Then the semilattices $\operatorname{Con}_{\mathrm{c}} \mathcal{L}(R)$ and $\operatorname{Id}_{\mathrm{c}} R$ (the semilattice of finitely generated two-sided ideals of $R$ ) are isomorphic (distributive) semilattices.

A matricial algebra over a field $F$ is a finite direct product of full matricial algebras over $F$. A direct limit of matricial algebras over $F$ is called a locally matricial algebra over $F$. Locally matricial algebras are regular. If $F$ is a finite field and $R$ is a locally matricial algebra over $F$, then $R$ is a locally finite ring and $\mathcal{L}(R)$ is a locally finite lattice. The following theorem appears first in well-known unpublished notes of G. M. Bergman [1]:

Theorem 7.3. Let $F$ be a field. Then every countable distributive $\langle\vee, 0\rangle$-semilattice is isomorphic to $\operatorname{Id}_{\mathrm{c}} R$, for some locally matricial algebra $R$ over $F$.

P. Růžička proves in [40] by a very sophisticated construction that Bergman's theorem holds also for distributive lattices of arbitrary cardinality.

Theorem 7.4. Let $F$ be a field. Then every distributive lattice with zero is isomorphic to $\operatorname{Id}_{\mathrm{c}} R$, for some locally matricial algebra $R$ over $F$.

The problem whether every distributive semilattice of cardinality $\aleph_{1}$ is isomorphic to $\operatorname{Id}_{\mathrm{c}} R$, for some locally matricial ring $R$, remains open, see Problem 3 . Because of the following result in F. Wehrung [49], the cardinality $\aleph_{1}$ is the maximal cardinality for which there could be a general positive answer:

Theorem 7.5. There exists a distributive semilattice of cardinality $\aleph_{2}$ that is not isomorphic to $\operatorname{Id}_{\mathrm{c}} R$, for any regular ring $R$.

On the other hand, every distributive $\langle\vee, 0\rangle$-semilattice of cardinality $\aleph_{1}$ can be represented as $\operatorname{Id}_{\mathrm{c}} R$, for some regular ring $R$, as also proved in F. Wehrung [51]. In the spirit of Problem 3, we can also mention the following difficult one-dimensional analogue of Theorem 7.3, established in J. Tůma and F. Wehrung [47]:

Theorem 7.6. Let $F$ be a field, let $S$ and $T$ be countable distributive $\langle\vee, 0\rangle$-semilattices, let $\varphi: S \rightarrow T$ be a $\langle\vee, 0\rangle$-homomorphism. Then there are locally matricial algebras $A$ and $B$ over $F$, a homomorphism $f: A \rightarrow B$ of $F$-algebras, and isomorphisms $\alpha: \operatorname{Id}_{\mathrm{c}} A \rightarrow S$ and $\beta: \operatorname{Id}_{\mathrm{c}} B \rightarrow T$ such that $\beta \circ \operatorname{Id}_{\mathrm{c}} f=\varphi \circ \alpha$.

An interesting point about the proof of Theorem 7.6 is that it involves a reverse one-dimensional amalgamation result. We say that a partially ordered vector space (over the field $\mathbb{Q}$ of rational numbers) is simplicial, if it is isomorphic to a finite power of $\mathbb{Q}$ with componentwise ordering, and that it is a dimension vector space, if it is isomorphic to a direct limit of simplicial vector spaces. The main result in J. Tůma and F. Wehrung [47] is that for a countable dimension vector space $V$ and a countable distributive $\langle\vee, 0\rangle$-semilattice $S$, every $\langle\vee, 0\rangle$-homomorphism from $S$ to $\operatorname{Id}_{\mathrm{c}} V$ can be lifted by a positive homomorphism from $U$ to $V$, for some (countable) dimension vector space $U$. The final step from dimension groups to locally matricial algebras uses results from K. R. Goodearl and D. E. Handelman [7].

Theorem 7.6 yields the following lattice-theoretical consequence: 
Corollary 7.7. Let $S$ and $T$ be countable distributive $\langle\vee, 0\rangle$-semilattices, let $\varphi: S \rightarrow T$ be a $\langle\vee, 0\rangle$-homomorphism. Then there are locally finite, relatively complemented modular lattices $K$ and $L$, a lattice homomorphism $f: K \rightarrow L$, and isomorphisms $\alpha: \operatorname{Con}_{\mathrm{c}} K \rightarrow S$ and $\beta: \operatorname{Con}_{\mathrm{c}} L \rightarrow T$ such that $\beta \circ \operatorname{Con}_{\mathrm{c}} f=\varphi \circ \alpha$.

It is also established in J. Tưma and F. Wehrung [47] that the analogue of Corollary 7.7 for $S$ uncountable fails.

\section{Dual topological spaces}

In the important papers [36] and [37], M. Ploščica investigates dual topological spaces of some congruence lattices. Any algebraic distributive lattice $D$ defines a topological space $\mathrm{M}(D)$. The points of $\mathrm{M}(D)$ are completely meet-irreducible elements of $D$ and closed sets of $\mathrm{M}(D)$ are sets of the form $\mathrm{M}(D) \cap\left[x, 1_{D}\right]$, for any $x \in X$. The lattice $D$ can be reconstructed from its dual space $\mathrm{M}(D)$ as the lattice of open subsets of $\mathrm{M}(D)$ ordered by inclusion.

If $L$ is a lattice, then the points of the dual space $\mathrm{M}(\operatorname{Con} L)$ of the full congruence lattice of $L$ are the subdirectly irreducible congruences of $L$, that is, the congruences $\boldsymbol{a}$ of $L$ such that the quotient lattice $L / \boldsymbol{a}$ is subdirectly irreducible. It seems that the dual spaces $\mathrm{M}(\operatorname{Con} L)$ might be a useful tool in the study of the congruence lattices of members of lattice varieties with only finitely many non-isomorphic subdirectly irreducible lattices.

The dual spaces $\mathrm{M}(D)$ have a base of compact open sets but they are not usually Hausdorff.

Definition 8.1. For a class $\mathbf{C}$ of lattices, we define

$$
\text { Con } \mathbf{C}=\{D \mid D \cong \text { Con } L \text {, for some } L \in \mathbf{C}\},
$$

the congruence class of $\mathbf{C}$.

M. Ploščica proves in [36] that the congruence classes Con $\mathbf{M}_{n}$ (here $\mathbf{M}_{n}$ denotes the variety generated by $M_{n}$, the lattice of length two with $n+2$ elements) are distinct. The topological property that distinguishes them is uniform separability.

Definition 8.2. A subset $Q$ of a topological space $T$ is called discrete, if every subset of $Q$ is open in the relative topology on $Q$. The space $T$ is called uniformly $n$-separable (for $n \geq 3$ ), if for every discrete set $Q \subseteq T$, there exists a family $\left\langle U_{p q} \mid p, q \in Q, p \neq q\right\rangle$ of open sets such that $p \in U_{p q}$, for every $p, q \in Q$, and, for every $n$-element set $Q_{0} \subseteq Q$,

$$
\bigcap\left\{U_{p q} \mid p, q \in Q_{0}, p \neq q\right\}=\varnothing .
$$

The following two theorems establish the crucial separability properties of the spaces $\mathrm{M}(\mathrm{Con} L)$, for $L \in \mathbf{M}_{n}$.

Theorem 8.3. If $L \in \mathbf{M}_{n}, n \geq 3$, then $\mathrm{M}(\operatorname{Con} L)$ is $(n+1)$-uniformly separable.

In order to prove Theorem 8.3, M. Ploščica [36] assumes that $(n+1)$-uniform separability fails in $\mathrm{M}(\mathrm{Con} L)$, and infers, with the help of a clever combinatorial statement, that $M_{n}$ has $n+1$ distinct atoms, a contradiction. 
Let $F_{n}(X)$ denote the free lattice over $X$ in the variety $\mathbf{M}_{n}$, for $n \geq 3$ and any set $X$

Theorem 8.4. The topological space $\mathrm{M}\left(\operatorname{Con} F_{n}(X)\right)$ is not n-uniformly separable.

As the proof of Theorem 2.6(ii) is based on the Kuratowski Free Set Theorem, the proof of Theorem 8.4 is based on the following extension of that theorem, due to A. Hajnal and A. Máté [31]:

Theorem 8.5. Let $X$ be a set of cardinality at least $\aleph_{2}$, let $\Phi:[X]^{2} \rightarrow[X]<\omega$. Then for every natural number $n \geq 3$, there exists $U \in[X]^{n}$ such that $u \notin \Phi(V)$, for all $u \in U$ and all $V \in[U \backslash\{u\}]^{2}$.

As a corollary, we get the following:

Corollary 8.6. Let $n \geq 3$, let $X$ be a set of cardinality at least $\aleph_{2}$. Then there is no lattice $L \in \mathbf{M}_{n}$ such that Con $L$ is isomorphic to $\operatorname{Con} F_{n+1}(X)$.

In his other paper [37], M. Ploščica characterizes dual spaces $\mathrm{M}(\operatorname{Con} L)$, for lattices $L$ with at most $\aleph_{1}$ compact elements from the variety $\mathbf{M}_{n}^{01}$ generated by $M_{n}$ as a bounded lattice, $n \geq 3$. His main result is the following deep theorem:

Theorem 8.7. Let $D$ be an algebraic distributive lattice with at most $\aleph_{1}$ compact elements and $n \geq 3$. Then $D$ is isomorphic to Con $L$, for some $L \in \mathbf{M}_{n}^{01}$, if and only if the topological space $T=\mathrm{M}(D)$ has a subspace $T_{0}$ that satisfies the following five conditions:

(i) $T$ is compact and has a basis of compact open sets;

(ii) both $T_{0}$ and $T_{n}=T \backslash T_{0}$ are Hausdorff zero-dimensional;

(iii) $T_{0}$ is a closed subspace of $T$;

(iv) if $a \in T_{n}, b \in T \backslash\{a\}$, then there exists a clopen set $V \subseteq T_{n}$ such that $a \in V$ and $b \notin V$;

(v) if $a, b, c \in T$ are distinct, then there exist open sets $U, V, W$ such that $a \in U, b \in V, c \in W$, and $U \cap V \cap W=\varnothing$.

In order to establish the harder direction of Theorem 8.7, M. Ploščica embeds directly, via an elaborate ad hoc construction, the space $T$ as a closed subspace of $\mathrm{M}\left(\operatorname{Con}\left(F_{n}^{01}\left(\omega_{1}\right)\right)\right)$, where $F_{n}^{01}\left(\omega_{1}\right)$ denotes the free object on $\aleph_{1}$ generators in the variety $\mathbf{M}_{n}^{01}$.

Since the conditions on $\mathrm{M}(D)$ do not depend on $n \geq 3$, we get the following corollary, see M. Ploščica [37].

Corollary 8.8. If $L \in \mathbf{M}_{n}^{01}, n \geq 3$, and $L$ has at most $\aleph_{1}$ elements, then there exists $K \in \mathbf{M}_{3}^{01}$ such that Con $K$ is isomorphic to Con $L$.

These results further emphasize the crucial role that the cardinality $\aleph_{2}$ plays in the study of congruence lattices of lattices; see also Problem 5.

\section{Open problems}

There are many open problems related to CLP, scattered in the literature. Here are, to our minds, the most outstanding ones. 
We first restate Dilworth's still unsolved problem:

Congruence Lattice Problem. Let $S$ be a distributive $\langle\vee, 0\rangle$-semilattice. Does there exist a lattice $L$ such that $\operatorname{Con}_{\mathrm{c}} L \cong S$ ?

Our next problem is a byproduct of the study of 1-CLP:

Problem 1. Let $K$ be a countable lattice, let $S$ be a countable distributive $\langle\vee, 0\rangle$ semilattice, let $\varphi$ : $\operatorname{Con}_{\mathrm{c}} K \rightarrow S$ be a $\langle\vee, 0\rangle$-homomorphism. Can $\varphi$ be lifted, that is, are there a lattice $L$, a lattice homomorphism $f: K \rightarrow L$, and an isomorphism $\varepsilon: \operatorname{Con}_{\mathrm{c}} L \rightarrow S$ such that $\varepsilon \circ \operatorname{Con}_{\mathrm{c}} f=\varphi$ ?

We observe that the cardinality assumption on $K$ and $S$ in Problem 1 is optimal. Indeed, the paper J. Tůma and F. Wehrung [46] contains an example of a $\langle\vee, 0\rangle$-homomorphism $\varphi: \operatorname{Con}_{\mathrm{c}} B \rightarrow S$, where $B$ is a Boolean lattice of cardinality $\aleph_{1}$ and $S$ is a countable distributive $\langle\vee, 0\rangle$-semilattice, that cannot be lifted. We also observe that the analogue of Problem 1, where $K$ is only a partial lattice, fails, because of some results in F. Wehrung [53]. Nevertheless, we still conjecture that Problem 1 has a positive solution.

By Schmidt's Lemma (see Theorem 6.6), every distributive image of a generalized Boolean semilattice is representable. This suggests the following problem:

Problem 2. Let $K$ be a lattice, let $S$ be a distributive $\langle\vee, 0\rangle$-semilattice, let $\varphi: \operatorname{Con}_{\mathrm{c}} K \rightarrow S$ be a surjective distributive $\langle\vee, 0\rangle$-homomorphism. Can $\varphi$ be lifted?

Of course, if $\varphi$ can be lifted, then the semilattice $S$ is representable. Problem 2 is first stated in J. Tůma and F. Wehrung [46, Problem 2].

Problem 3. Is it the case that every distributive $\langle\vee, 0\rangle$-semilattice of cardinality $\aleph_{1}$ is isomorphic to $\operatorname{Con}_{\mathrm{c}} L$, for some sectionally complemented, modular, locally finite lattice $L$ ?

A ring-theoretical equivalent to Problem 3 is whether every distributive $\langle\vee, 0\rangle$ semilattice of cardinality $\aleph_{1}$ is isomorphic to $\operatorname{Id}_{\mathrm{c}} R$, for some locally matricial algebra $R$, see K. R. Goodearl and F. Wehrung [8]. The countable case is solved by Bergman's Theorem, see Theorem 7.3. The statement obtained by removing "locally finite" from the statement of Problem 3 is proved in F. Wehrung [51]. The statement obtained by removing "modular" from the statement of Problem 3 is proved in G. Grätzer, H. Lakser, and F. Wehrung [10]. Various aspects and possible attacks of Problem 3 are also studied in J. Tůma and F. Wehrung [47].

A problem related to Problem 1 is the following:

Problem 4. Does every lattice of cardinality at most $\aleph_{0}$ (resp., $\aleph_{1}$ ) have a congruence-preserving extension to a relatively complemented lattice?

It is proved in G. Grätzer and E. T. Schmidt [14] that every finite lattice has a finite, sectionally complemented congruence-preserving extension. Further results imply that every lattice $L$ in each of the following classes has a relatively complemented congruence-preserving extension that it generates as a convex sublattice:

- $L$ is a direct union $\bigcup_{n<\omega} L_{n}$, where $\operatorname{Con}_{\mathrm{c}} L_{n}$ is finite, for all $n<\omega$ (G. Grätzer, H. Lakser, and F. Wehrung [10]). 
- $L$ is a direct union $\bigcup_{n<\omega} L_{n}$, where $\operatorname{Con}_{\mathrm{c}} L_{n}$ is conditionally co-Brouwerian, for all $n<\omega$ (F. Wehrung [53]).

- $\mathrm{Con}_{\mathrm{c}} L$ is a lattice (F. Wehrung [52]).

On the other hand, the cardinality $\aleph_{1}$ in the statement of Problem 4 is the highest possible, because of the results of M. Ploščica, J. Tůma, and F. Wehrung [38] and J. Tůma and F. Wehrung [45]. For example, for any nondistributive variety $\mathbf{V}$ of lattices, the free lattice in $\mathbf{V}$ on $\aleph_{2}$ generators does not have a congruence-preserving extension with permutable (or even almost permutable) congruences.

For varieties $\mathbf{U}$ and $\mathbf{V}$ of lattices, define the critical point of $\mathbf{U}$ and $\mathbf{V}$ as the least cardinality of the semilattice of compact elements of a member of the symmetric difference $($ Con $\mathbf{U}) \triangle($ Con $\mathbf{V})$ (let it be $\infty$, if Con $\mathbf{U}=\operatorname{Con} \mathbf{V})$.

Problem 5 (Critical point conjecture). Let $\mathbf{U}$ and $\mathbf{V}$ be varieties of lattices (resp., finitely generated varieties of lattices), with critical point $\kappa<\infty$. Prove that either $\kappa \leq \aleph_{0}$ or $\kappa=\aleph_{2}$.

In all known cases, the answer to Problem 5 is positive. Even for finitely generated varieties, this problems seems to be very difficult.

For a lattice $L$, let $\operatorname{Var} L$ denote the lattice variety generated by $L$, and $\operatorname{Con} \operatorname{Var} L$ its congruence class (see Definition 8.1). We do know whether, for finite lattices $A$ and $B$, the equality $\operatorname{Con} \operatorname{Var} A=\operatorname{Con} \operatorname{Var} B$ can be checked recursively. In particular, the following sounds plausible:

Problem 6. For finite lattices $A$ and $B$, does Con Var $A=$ Con Var $B$ imply that either $A \cong B$ or $A \cong B^{\mathrm{d}}$ ?

It follows from M. Ploščica's results [36] that the congruence classes Con $\mathbf{M}_{n}$, for $n \geq 3$, are distinct, see Section 8 . However, they can be separated only by semilattices of cardinality at least $\aleph_{2}$, see Corollary 8.8.

Problem 7. Characterize the congruence classes of the varieties $\mathbf{M}_{n}$, for $n \geq 3$.

Up to now, the only nontrivial variety of which the congruence class is completely described is the variety of distributive lattices, for which the congruence class is the class of all lattices of ideals of generalized Boolean semilattices (this is trivial and well-known). Necessary conditions for a given distributive $\langle\vee, 0\rangle$-semilattice to belong to the congruence class of $\mathbf{M}_{n}$ are given in Theorems 8.3 and 8.7.

Problem 8. Are the congruence classes of sectionally complemented and relatively complemented lattices distinct?

Of course, further variants of Problem 8 could be stated for other classes of lattices, for example, the class of sectionally complemented modular lattices or the class of lattices with permutable congruences. A basic approach for solving Problem 8 is the following:

Problem 9. For a sectionally complemented lattice $L$, does $\mathrm{Con}_{\mathrm{c}} L$ satisfy $\mathrm{URP}^{+}$?

We proved in Proposition 2.10 that if $L$ is relatively complemented, then $\operatorname{Con}_{\mathrm{c}} L$ satisfies $\mathrm{URP}^{+}$, but the argument fails for sectionally complemented lattices. 
Problem 10. Let $S$ be a distributive $\langle\vee, 0\rangle$-semilattice that is the image of a generalized Boolean semilattice under a distributive homomorphism. Does $S$ satisfy $\mathrm{URP}^{+}$?

Problem 11. Is the property URP preserved under tensor product (resp., iterated tensor product) of $\langle\vee, 0\rangle$-semilattices?

Finally we observe that the problem about which semilattices $S$ satisfy 1-CLP (see Definition 5.3) is completely solved in F. Wehrung [52] and J. Tůma and F. Wehrung [46]: namely, these are exactly the distributive lattices with zero. The two-dimensional analogue 2-CLP is not completely solved yet:

Problem 12. Let $S$ be a distributive $\langle\vee, 0\rangle$-semilattice. Does any of the following assumptions imply that $S$ is conditionally co-Brouwerian:

(i) For every partial lattice $P$, every $\langle\vee, 0\rangle$-homomorphism $\varphi$ : $\operatorname{Con}_{\mathrm{c}} P \rightarrow S$ can be factored through a lattice;

(ii) For every partial lattice $P$, every $\langle\vee, 0\rangle$-homomorphism $\varphi$ : $\operatorname{Con}_{\mathrm{c}} P \rightarrow S$ can be lifted through a lattice;

(iii) For every truncated square $\mathcal{D}$ of lattices, every $\langle\vee, 0\rangle$-homomorphism $\varphi: \mathrm{Con}_{\mathrm{c}} \mathcal{D} \rightarrow S$ can be factored through a lattice;

(iv) For every truncated square $\mathcal{D}$ of lattices, every $\langle\vee, 0\rangle$-homomorphism $\varphi: \mathrm{Con}_{\mathrm{c}} \mathcal{D} \rightarrow S$ can be lifted through a lattice.

It is proved in F. Wehrung [53] that the assumption that $S$ be conditionally coBrouwerian is sufficient to imply (i)-(iv) above, see Theorem 6.4. Moreover, some partial converses to this statement are proved in F. Wehrung [53]: namely, either (i), (ii), or (iv) implies that $S$ is a conditionally complete lattice.

\section{Acknowledgments}

This work was partially completed while the second author was visiting the Charles University (Prague). Excellent conditions provided by the Department of Algebra are greatly appreciated. The authors also wish to thank Marina Semenova and George Grätzer for their comments.

\section{REFERENCES}

[1] G. M. Bergman, Von Neumann regular rings with tailor-made ideal lattices, Unpublished note (26 October 1986).

[2] R. A. Dean, Free lattices generated by partially ordered sets and preserving bounds, Canad. J. Math. 16 (1964), 136-148.

[3] R. P. Dilworth, Lattices with unique complements, Trans. Amer. Math. Soc. 57, no. 1 (1945), 123-154.

[4] R. Freese, J. Ježek, and J. B. Nation, "Free Lattices", Mathematical Surveys and Monographs, 42, Amer. Math. Soc., Providence, 1995. viii+293 p.

[5] K. D. Fryer and I. Halperin, The von Neumann coordinatization theorem for complemented modular lattices, Acta Sci. Math. (Szeged) 17 (1956), 203-249.

[6] N. Funayama and T. Nakayama, On the distributivity of a lattice of lattice congruences, Proc. Imp. Acad. Tokyo 18 (1942), 553-554. 
[7] K. R. Goodearl and D. E. Handelman, Tensor products of dimension groups and $K_{0}$ of regular rings, Canad. J. Math. 38, no. 3 (1986), 633-658.

[8] K. R. Goodearl and F. Wehrung, Representations of distributive semilattices in ideal lattices of various algebraic structures, Algebra Universalis 45 (2001), 71-102.

[9] G. Grätzer, "General Lattice Theory. Second edition", new appendices by the author with B. A. Davey, R. Freese, B. Ganter, M. Greferath, P. Jipsen, H. A. Priestley, H. Rose, E. T. Schmidt, S. E. Schmidt, F. Wehrung, and R. Wille. Birkhäuser Verlag, Basel, 1998. xx+663 p.

[10] G. Grätzer, H. Lakser, and F. Wehrung, Congruence amalgamation of lattices, Acta Sci. Math. (Szeged) 66 (2000), 339-358.

[11] G. Grätzer and E. T. Schmidt, Characterizations of congruence lattices of abstract algebras, Acta Sci. Math. (Szeged) 24 (1963), 34-59.

[12] _ A lattice construction and congruence-preserving extensions, Acta Math. Hungar. 66 (1995), 275-288.

[13] _ The Strong Independence Theorem for automorphism groups and congruence lattices of finite lattices, Beiträge Algebra Geom. 36 (1995), 97-108.

[14] _ Congruence-preserving extensions of finite lattices to sectionally complemented lattices, Proc. Amer. Math. Soc. 127 (1999), 1903-1915.

[15] _ Congruence Lattices, Appendix C in [9], 519-530.

[16] _ Regular congruence-preserving extensions of lattices, Algebra Universalis 46 (2001), $119-130$.

[17] _ Representing congruence lattices of lattices with partial unary operations as congruence lattices of lattices. I. Interval equivalence, J. Algebra, to appear.

[18] G. Grätzer and M. Greenberg, Lattice tensor products. I. Coordinatization, Acta Math. Hungar. 95 (4) (2002), 265-283.

[19] _ Lattice tensor products. II. Ideal lattices, Acta Math. Hungar. 97 (3) (2002), 179184.

[20] _ Lattice tensor products. III. Congruences, Acta Math. Hungar. 98 (1-2) (2003), 189-199.

[21] _ Lattice tensor products. IV. Infinite lattices, preprint.

[22] G. Grätzer, M. Greenberg, and E. T. Schmidt, Representing congruence lattices of lattices with partial unary operations as congruence lattices of lattices. II. Interval ordering, preprint.

[23] G. Grätzer, H. Lakser, and R.W. Quackenbush, The structure of tensor products of semilattices with zero, Trans. Amer. Math. Soc. 267 (1981), 503-515.

[24] G. Grätzer and E. T. Schmidt, Finite lattices and congruences. A survey, preprint.

[25] G. Grätzer and F. Wehrung, Proper congruence-preserving extensions of lattices, Acta Math. Hungar. 85 (1999), 169-179.

[26] 815.

[27] , The $M_{3}[D]$ construction and n-modularity, Algebra Universalis 41 (1999), 87-114.

[28] , Tensor products of lattices with zero, revisited, J. Pure Appl. Algebra 147 (2000), $273-301$.

[29] _ A new lattice construction: the box product, J. Algebra 221 (1999), 315-344.

[30] The Strong Independence Theorem for automorphism groups and congruence lattices of arbitrary lattices, Adv. in Appl. Math. 24 (2000), 181-221.

[31] A. Hajnal and A. Máté, Set mappings, partitions, and chromatic numbers, Logic Colloquium 1973 (Studies in Logic and the Foundations of Mathematics, Vol. 80), Proc. Bristol 1973, North-Holland, 347-379 (1975).

[32] A.P. Huhn, On the representation of algebraic distributive lattices II, Acta Sci. Math. (Szeged) 53 (1989), 3-10.

[33] - On the representation of algebraic distributive lattices III, Acta Sci. Math. (Szeged) 53 (1989), 11-18.

[34] C. Kuratowski, Sur une caractérisation des alephs, Fund. Math. 38 (1951), 14-17.

[35] J. von Neumann, On regular rings, Proc. Nat. Acad. Sci. USA 22 (1936), 707-713. 
[36] M. Ploščica, Separation properties in congruence lattices of lattices, Colloq. Math. 83 (2000), $71-84$.

[37] _ Dual spaces of some congruence lattices, Topology Appl., to appear.

[38] M. Ploščica, J. Tůma, and F. Wehrung, Congruence lattices of free lattices in nondistributive varieties, Colloq. Math. 76, no. 2 (1998), 269-278.

[39] P. Pudlák, On congruence lattices of lattices, Algebra Universalis 10 (1980), 74-95.

[40] P. Růžička, Lattices of two-sided ideals of locally matricial algebras and the $\Gamma$-invariant problem, preprint.

[41] E. T. Schmidt, Zur Charakterisierung der Kongruenzverbände der Verbände, Mat. Casopis Sloven. Akad. Vied 18 (1968), 3-20.

[42] _ The ideal lattice of a distributive lattice with 0 is the congruence lattice of a lattice, Acta Sci. Math. (Szeged) 43 (1981), 153-168.

[43] M. Tischendorf, The representation problem for algebraic distributive lattices, Ph.D. thesis, TH Darmstadt, 1992.

[44] J. Tůma, On the existence of simultaneous representations, Acta Sci. Math. (Szeged) 64 (1998), 357-371.

[45] J. Tůma and F. Wehrung, Simultaneous representations of semilattices by lattices with permutable congruences, Internat. J. Algebra Comput. 11, no. 2 (2001), 217-246.

[46] Unsolvable one-dimensional lifting problems for congruence lattices of lattices, Forum Math. 14, no. 4 (2002), 483-493.

[47] Liftings of diagrams of semilattices by diagrams of dimension groups, Proc. London Math. Soc., to appear.

[48] F. Wehrung, Injective positively ordered monoids I, J. Pure Appl. Algebra 83 (1992), 43-82.

[49] — Non-measurability properties of interpolation vector spaces, Israel J. Math. 103 (1998), 177-206.

[50] _ A uniform refinement property for congruence lattices, Proc. Amer. Math. Soc. 127, no. 2 (1999), 363-370.

[51] _ Representation of algebraic distributive lattices with $\aleph_{1}$ compact elements as ideal lattice of regular rings, Publ. Mat. 44 (2000), 419-435.

[52] _ Forcing extensions of partial lattices, J. Algebra, to appear.

[53] _ Join-semilattices with two-dimensional congruence amalgamation, Colloq. Math. 93, no. 2 (2002), 209-235.

Department of Algebra, Faculty of Mathematics and Physics, Sokolovská 83, Charles University, 18600 Praha 8, Czech Republic

E-mail address: tuma@karlin.mff.cuni.cz

CNRS, UMR 6139, Département de Mathématiques, Université de Caen, 14032 Caen Cedex, France

E-mail address: wehrung@math.unicaen.fr

$U R L:$ http://www.math.unicaen.fr/ wehrung 